Accepted refereed manuscript of:

Hoyle A \& Bowers R (2008) Can possible evolutionary outcomes be determined directly from the population dynamics?, Theoretical Population Biology, 74 (4), pp. 311-323.

DOI: $\underline{10.1016 / j . t p b .2008 .09 .002}$

(C) 2008, Elsevier. Licensed under the Creative Commons AttributionNonCommercial-NoDerivatives 4.0 International http://creativecommons.org/licenses/by-nc-nd/4.0/ 


\title{
Can possible evolutionary outcomes be determined directly from the population dynamics?
}

\author{
Andrew Hoyle ${ }^{*}$, Roger G. Bowers ${ }^{2}$ \\ ${ }^{1}$ Department of Computing Science and Mathematics, University of Stirling, \\ Stirling, FK9 4LA, U.K. \\ ${ }^{2}$ Department of Mathematical Sciences, Mathematical Sciences Building, The \\ University of Liverpool, Liverpool, L69 7ZL, U.K.
}

\begin{abstract}
Traditionally, to determine the possible evolutionary behaviour of an ecological system, using adaptive dynamics, it is necessary to calculate the fitness and its derivatives at a singular point. We investigate the claim that the possible evolutionary behaviour can be predicted directly from the population dynamics, without the need for calculation, by applying three criteria - one based on the form of the density dependent rates and two on the role played by the evolving parameters. Taking a general continuous time model, with broad ecological range, we show that the claim is true. Initially, we assume that individuals enter in class 1 and move through population classes sequentially; later we relax these assumptions and find that the criteria still apply. However, when we consider models where the evolving parameters appear non-linearly in the dynamics, we find some aspects of the criteria fail; useful but weaker results on possible evolutionary behaviour now apply.
\end{abstract}

Key words: Adaptive dynamics, trade-off and invasion plot, TIPs, trade-off, 
invasion boundaries, evolutionary branching.

* Corresponding author. Tel.: +44-1786 467 467; fax: +44-1786 464551.

Email address: A.Hoyle@maths.stir.ac.uk (Andrew Hoyle ${ }^{1}$ ). 


\section{Introduction}

It has long been recognised that trade-offs play an important role in evolutionary theory (see Stearns (1992) and Roff (2002) for reviews). However it is only relatively recently that evolutionary ecologists have come to realise the extent of this role and how minor changes in a trade-off, for example in its shape, can dramatically affect the evolutionary outcome in a system (Levins, 1962, 1968; Rueffler et al., 2004; De Mazancourt and Dieckmann, 2004; Bowers et al., 2005). In early work, the picture that emerged seems often to be that acceleratingly costly trade-offs (where each benefit is met with an ever increasing cost) produced an intermediate state via an evolutionary attractor (or CSS), whereas deceleratingly costly trade-offs (where each benefit is met with an ever decreasing cost) produced an extreme state via an evolutionary repellor (Levins, 1962). More recent work using the general framework of adaptive dynamics (Metz et al., 1996a; Geritz et al., 1998) - which stresses the importance of density-dependent feedback - has shown that the above picture is not generally valid (Rueffler et al., 2004; De Mazancourt and Dieckmann, 2004; Bowers et al., 2005; see also Bowers et al. (2003) for an informative example).

The traditional approach to adaptive dynamics tends to be rather algebraic although it does have a geometrical side (pairwise invadability plots, or PIPs) and to 'bury' the trade-off in a way not facilitating further study. In the present context recent geometrical approaches to adaptive dynamics are of great utility (Rueffler et al., 2004; De Mazancourt and Dieckmann, 2004; Bowers et al., 2005). These geometrical approaches keep the trade-off at the forefront of the work and allow more informative, graphical conclusions to be drawn regarding 
which trade-off shapes produce which evolutionary outcome. Here by 'shape' we mean whether a trade-off is acceleratingly costly or deceleratingly costly (or linear - where each benefit is met with the same cost) and by what magnitude or strength. There have been a number of studies into the evolutionary outcomes of various ecological systems using these new geometric approaches (see references above and Geritz et al., 2007; Hoyle and Bowers, 2007). A common feature of these studies is that strongly acceleratingly costly trade-offs lead to intermediate traits (evolutionary attractors) and strongly deceleratingly costly trade-offs lead to extreme traits (evolutionary repellors); what happens between these, for weakly acceleratingly/deceleratingly costly tradeoffs, and even linear trade-offs, is less clear - although branching points or Garden of Eden points (ESS-repellors) (Metz et al., 1996a; Geritz et al., 1998) may appear. The comparative ease by which the new geometrical approaches have allowed the study of various specific systems is notable. Thus our aim here is to investigate a relatively general model, that covers behaviour such as maturation, competition, predation and parasitism, in order to elucidate the factors in the trade-off and in the dynamics of the model which give rise to various evolutionary outcomes.

The geometrical method we will use in this study is that of trade-off and invasion plots (TIPs) (Bowers et al., 2005). Although a detailed discussion of TIPs can be found in Bowers et al. (2005), we give here, and in Appendix A, a summary of TIPs and the major results determining evolutionary behaviour from these. A TIP is a plot between two traits of one strain, $y$ say (with traits $y_{1}$ and $y_{2}$ ), where the second strain, $x$ say (with traits $x_{1}$ and $x_{2}$ ), is taken to be fixed, i.e. a fixed point on the plot (usually taken at a corner). Underlying a TIP is the fitness $s_{x}(y)$ of a rare mutant strain $y$ when the resident is $x$. 
These plots then consist of two invasion boundaries (curves), one of which, $f_{1}$ (which is equivalent to $s_{x}(y)=0$ ), defines where one strain (when rare), $y$, can invade a second (established) strain, $x$, and the second of which, $f_{2}$ (which is equivalent to $s_{y}(x)=0$ ), defines where (rare) strain $x$ can invade (established) strain $y$ (when the roles are reversed). Both of these invasion boundaries are equal and tangential at the point $y=x$. The third curve on a TIP is the trade-off curve, $f$. This is equal to $f_{1}$ and $f_{2}$ at the point $y=x$ and, for certain $x$, is also tangential to the invasion boundaries; these $x$ are the evolutionary singularities, $x^{*}$. It is the relative curvatures (or shapes) of the three curves at the singularity that determine the evolutionary outcome. The invasion boundaries determine which evolutionary outcomes are possible, whereas the trade-off determines which actually occurs. For more details on TIPs see Appendix A and Bowers et al. (2005).

As stated above, the invasion boundaries, near the evolutionary singularity, determine which evolutionary outcomes are possible. The mutational steps are sufficiently small that it is the local behaviour of these invasion boundaries (and of the trade-off) up to quadratic approximation which we invariably describe (we avoid continually repeating this point). Subject to this, there are four fundamental types of (singular) TIP each with their own unique evolutionary possibilities. These are shown in Fig. 1. We call the first a type I TIP; here the invasion boundaries are both linear and superimposed. With this type of TIP only evolutionary attractors (for acceleratingly costly trade-offs) and evolutionary repellors (for deceleratingly costly trade-offs) are possible. This represents the picure that emerged in early work (Levins, 1962). The second type of TIP is type II; here the invasion boundaries are again superimposed but are now curved. Again only evolutionary attractors and repellors are pos- 
sible, but now for weakly deceleratingly costly trade-offs attractors can replace repellors or for weakly acceleratingly costly trade-offs repellors can replace attractors. In type III and type IV TIPs, the invasion boundaries are no longer superimposed. In type III one boundary is linear with the second boundary curved, whereas in type IV both invasion boundaries are curved. The separation of boundaries has significant implications for the evolutionary outcomes. For certain trade-offs with curvature between the invasion boundaries either evolutionary branching points or Garden of Eden points (ESS-repellors) may be possible (which of these are possible depends upon the relative curvatures of $f_{1}$ and $f_{2}$; see Table A.1).

So the question arises as to what factors determine the shape of the invasion boundaries and hence the type of TIP produced and evolutionary outcomes possible. Is it possible to establish criteria - which are necessary for various types of TIPs and the associated evolutionary possibilities - criteria which can be applied directly, without the need for further calculation, to the dynamical specification of a broad class of models in population ecology? This is the focus of the present paper.

\section{The three criteria}

We assume that time is continuous, that individuals can only be in a finite number of classes (or $i$-states), and that the number of individuals is sufficiently large that a deterministic approximation can be used. We take the dynamics to be given by the system of ordinary differential equations

$$
\frac{d \mathbf{N}}{d t}=\mathbf{M}(\mathbf{p}(\mathbf{x}), \mathbf{N}) \mathbf{N}
$$


the components $N_{i}$ of the $m$-dimensional column vector $\mathbf{N}$ are the densities of individuals in the various classes $i$, the matrix elements $M_{i j}$ are the rates of increase of $N_{i}$ per capita of class $j, \mathbf{p}$ is a vector of parameters and $\mathbf{x}=\left(x_{1}, x_{2}\right)$ is a trait vector which changes as the species evolves. Later we shall use the notation

$$
\begin{aligned}
& \mathbf{M}_{i \cdot}=\left(\begin{array}{lll}
M_{i 1} & \ldots & M_{i m}
\end{array}\right), \\
& \mathbf{M}_{\cdot j}=\left(\begin{array}{lll}
M_{1 j} & \ldots & M_{m j}
\end{array}\right),
\end{aligned}
$$

to denote the $i^{\text {th }}$ row and $j^{\text {th }}$ column respectively; we shall also abuse notation by writing $\mathbf{M}(\mathbf{x}, \mathbf{N})$ rather than the full form in Eq. (1).

We distinguish terms contributing to the matrix elements as follow

$$
M_{i j}=b_{i j}-\delta_{i j} e_{i j}-\delta_{i j} \sum_{k} t_{k i}+t_{i j}
$$

The first term $b_{i j}$, defined as a reproduction term, corresponds to the reproduction (birth) rate in class $i$ per capita of class $j$. Note that, if we take frequency dependent competition to act upon births, then this frequency dependence will appear in the corresponding reproduction term. The second term $e_{i i}$, defined as a mortality term, corresponds to the mortality per capita of class $i$. The third term $t_{i j}$, defined as a transition term, corresponds to the transition of individuals from class $j$ into class $i$ per capita of class $j$. The term $\delta_{i j}$ is the Kronecker delta. As indicated in Eq. (1) the matrix elements $M_{i j}$ and the individual terms underlying them can depend on the densities $\mathbf{N}$ and the trait $\mathrm{x}$.

Throughout we take one evolving species, made up of $n$ classes, possibly in the presence of a number of non-evolving species, each of which may consist 
of multiple classes. The matrix $\mathbf{M}$ is therefore split into a number of square sub-matrices, one for each species. The size of each sub-matrix is determined by the number of classes the species is described by (e.g. a species of 3 classes will be described by a $3 \times 3$ sub-matrix) and where the main diagonal of each sub-matrix lies on the main diagonal of the matrix $\mathbf{M}$. All remaining entries of $\mathbf{M}$, outside these sub-matrices, are zero. For the purposes of this study, we define the matrix $\mathbf{M}^{(n)}$ as the $n \times n$ sub-matrix describing the evolving species (made up of $n$ classes).

Hoyle et al. (2008) hypothesised three criteria and claimed that their occurrence in various combinations leads to a classification of possible TIPs. These criteria were suggested on the basis of the study of a very limited number of specific models. Here we prove their appropriateness for a relatively broad classes of ecological dynamics. (We impose some restrictions at first - but then lift many of them.)

The three criteria, which are based upon the dynamics describing the behaviour of the model, are:

- Criterion A: The Appearing Criterion

At least two of the row rate vectors $\mathbf{M}_{i}$. are (non-constant) functions of the trait vector $\mathbf{x}$; that is

$$
\mathbf{M}_{i \cdot}(\mathbf{x}, \mathbf{N}) \neq \mathbf{M}_{i \cdot}\left(\mathbf{x}^{\prime}, \mathbf{N}\right)
$$

for all $\mathbf{x}, \mathbf{x}^{\prime}$ for at least two values of $i$. (Consequently, a $t_{i j}$ and/or $b_{i j}$ and/or $e_{i j}$ must vary for at least two values of $i$, as $\mathbf{x}$ varies.) More informally, the evolving parameters, or one repeated evolving parameter, appear in the population dynamics of different classes/species.

- Criterion B: The Characteristic Criterion 
At least two of the column rate vectors $\mathbf{M}_{\cdot j}^{(n)}$ are (non-constant) functions of the trait vector $\mathbf{x}$; that is

$$
\mathbf{M}_{\cdot j}^{(n)}(\mathbf{x}, \mathbf{N}) \neq \mathbf{M}_{\cdot j}^{(n)}\left(\mathbf{x}^{\prime}, \mathbf{N}\right)
$$

for all $\mathbf{x}, \mathbf{x}^{\prime}$ for at least two values of $j$. More informally, the evolving parameters, or one evolving parameter alone, are characteristic of more than one class of the evolving species. (We say a parameter is characteristic of a class $j$ if $M_{i j}$, and consequently a $b_{i j}$ and/or $e_{i j}$ and/or $t_{i j}$, varies as the parameter varies.)

- Criterion C: The Rates Criterion.

For each $\mathbf{x}$, the image set of the map $\mathbf{N} \rightarrow M^{(n)}(\mathbf{x}, \mathbf{N})$ is at least two dimensional. More informally, at least two $M_{i j}^{(n)}$, or two components of the same $M_{i j}^{(n)}$ (and consequently two rates $b_{i j}$ and/or $e_{i j}$ and/or $t_{i j}$, or two components of the same rate) depend on densities differently.

For a relatively broad class of models, the effects of each of these criteria occuring in various combinations are claimed to be as in Table 1. See Fig. 1 for associated TIP types.

Some clarification is needed here, which we give by means of an example. Consider a simple host-pathogen SI model where the infected individuals neither reproduce nor recover. Here the dynamics are

$$
\frac{d \mathbf{X}}{d t}=\left(\begin{array}{cc}
a_{x}-q_{x} X_{1}-c_{x}-\beta_{x} X_{1} & \gamma_{x} \\
\beta_{x} X_{1} & c_{x}+\alpha_{x}+\gamma_{x}
\end{array}\right) \mathbf{X}
$$

Here $\mathbf{X}=\left(X_{1}, X_{2}\right)^{T}$ where $X_{1}$ and $X_{2}$ represent the densities of susceptible and infected individuals respectively. For the parameters, $a_{x}$ is the birth rate 
of susceptibles where we take density dependence to act upon the births at a rate $q_{x} ; b_{11}=a_{x}-q_{x} X_{1}$ and $b_{12}=b_{21}=b_{22}=0$. The (natural) death rate of susceptibles is $c_{x}$ and for infecteds is $c_{x}+\alpha_{x}$ (where $\alpha_{x}$ is the pathogen induced death rate), hence $e_{11}=c_{x}$ and $e_{22}=c_{x}+\alpha_{x}$. Finally $\beta_{x}$ is the transmission rate of the pathogen and $\gamma_{x}$ is the host recovery rate, hence $t_{21}=\beta_{x} X_{2}$ and $t_{12}=\gamma_{x}$. By considering the $q_{x} X_{1}$ and $\beta_{x} X_{2}$ terms, we can immediately say that criterion $\mathrm{C}$ is satisfied as these terms are affected differently by the population densities. We note that if we remove the density dependence on births, i.e. set $q_{x}=0$, then criterion $\mathrm{C}$ would no longer be satisfied. Let us consider three cases (assuming $q_{x} \neq 0$ ) regarding which parameters are evolving (those involved in the trade-off). Firstly, considering $a_{x}$ and $q_{x}$, both are contained in $M_{11}\left(\right.$ in $\left.b_{11}\right)$ only, hence neither criteria A or B is satisfied; here a type I TIP would occur (see Fig. 1). Secondly, considering $a_{x}$ and $\beta_{x}$, in combination these occur in $M_{11}$ (in $b_{11}$ and $t_{21}$ ) and $M_{21}$ (in $t_{21}$ ), thus criterion A is satisfied, as two rows of $M$ are affected, but criterion B is not; hence we can get a type III TIP. Finally, considering $a_{x}$ and $\alpha_{x}$, in combination these occur in $M_{11}$ (in $b_{11}$ ) and $M_{22}$ (in $e_{22}$ ), thus criteria A and $\mathrm{B}$ are both satisfied as they affect both rows and columns respectively; hence we can get a type IV TIP. Criterion B can only be satisfied if the dynamics of the evolving species are made up of more than one class; hence it cannot hold in, for example, classic predator-prey models where both predator and prey are made up of a single class (see for example, the models studied by Bowers and White (2002), White and Bowers (2005), Kisdi and Liu (2006) and Hoyle and Bowers (2007)).

The analysis we present below is framed in the context of continuous time models and restricted to the case where the system attains a stable fixed equilibrium before the next mutation step occurs. Initially, we impose the 
'technical' assumptions, typical of many such models, that all individuals are born into a single class and that they move through the classes in a particular order possibly returning to the initial class (which allows for recovery in infection models). Although this excludes models such as those in discrete-time or with other attractors such as cycles, it does cover models that are widely used (see, for example, Boots and Haraguchi, 1999; Dercole and Rinaldi, 2002; Boots and Bowers, 2004; Loeuille and Loreau, 2004; models based upon the SIS/SIR models founded by Anderson and May, 1979; and previous references). We thus take a general model for which these conditions hold and aim to prove that the combinations of the three criteria are necessary (although not sufficient) to give the appropriately shaped TIPs.

Although the assumptions do cover a broad class of systems, they can still be limiting. For example, they exclude vaccination in SIR models and infections with competing pathogens. For this reason, later work in this article will begin relaxing our 'technical' assumptions in order to see how generally our conclusions might apply.

One issue of broader importance deserves attention. In all of the analysis described above, we assume that the evolving parameters appear linearly in the dynamical equations and use these parameters as the coordinates for the construction of TIPs. (Intrinsic growth rates, transmission rates and recovery rates provide examples.) We give this point attention in a subsequent section and discuss the consequences for our work of adopting non-linear parameters and of smooth changes of coordinate system in the space of evolving parameters. 


\section{Proofs of the three criteria with the assumptions}

We recall the assumptions made earlier such that all individuals are born into class 1 - hence $b_{i j}=0$ if $i \neq 1$ - and move through the classes sequentially (possibly returning to the first class from the last) - hence only $t_{\{i+1\} i}$, for $i=1, \ldots, n-1$, and $t_{1 n}$ are non-zero.

The life cycle graph for the evolving species, consisting of 3 classes, is given in Fig. 2. Extending this to $n$ classes, the dynamics of the evolving species takes the form

$$
\begin{aligned}
\frac{d X_{1}}{d t} & =\sum_{j=1}^{n} \bar{b}_{1 j} X_{j}-\bar{e}_{11} X_{1}-\bar{t}_{21} X_{1}+\bar{t}_{1 n} X_{n}, \\
\frac{d X_{i}}{d t} & =\bar{t}_{i\{i-1\}} X_{i-1}-\bar{e}_{i i} X_{i}-\bar{t}_{\{i+1\} i} X_{i} \quad \text { for } i=2, . ., n,
\end{aligned}
$$

where $t_{\{n+1\} n}=t_{1 n}$.(Notice the use of per capita rates.) Here $X_{i}$ represents the density of the resident strain in class $i$, where $i=1, \ldots, n$, or a density of any non-evolving species, for $i=m-n+1, \ldots, m$, and $\bar{b}_{i j}, \bar{e}_{i i}$ and $\bar{t}_{i j}$ the rates of the resident strain where $\bar{b}_{i j}=B_{i j}\left(x, X_{1}(x), \ldots, X_{m}(x)\right)$ and similarly for the other rates. We take only one species to be evolving, possibly in the presence of a number of non-evolving species. However, for our present purposes we do not need to explicitly consider the equations describing the dynamics of any non-evolving species. In the calculation of the mutant fitness any interactions they have with the evolving species will be contained in the $\bar{b}_{i j}, \bar{e}_{i i}$ and $\bar{t}_{i j}$ terms.

If we introduce a mutation of resident strain $x$, denoted $y$, into this environment, and take it to be initially rare (at low density), then the dynamics can be written as 


$$
\begin{aligned}
& \frac{d Y_{1}}{d t}=\sum_{j=1}^{n} b_{1 j} Y_{j}-e_{11} Y_{1}-t_{21} Y_{1}+t_{1 n} Y_{n}, \\
& \frac{d Y_{i}}{d t}=t_{i\{i-1\}} Y_{i-1}-e_{i i} Y_{i}-t_{\{i+1\} i} Y_{i} \quad \text { for } i=2, . ., n .
\end{aligned}
$$

Here $Y_{i}$ denotes the density of the mutant invaders in class $i$. Since the mutant is rare we can assume the appropriate limits exist and ignore its densities in $b_{i j}, e_{i i}$ and $t_{i j}$; we thus have $b_{i j}=B_{i j}\left(y, X_{1}(x), \ldots, X_{m}(x)\right)$ and $\left.b_{i j}\right|_{y=x}=\bar{b}_{i j}$ etc and similarly for derivatives.

The fitness is defined as being the per capita growth rate of a rare mutant invader and is commonly denoted as $s_{x}(y)$, where $x$ denotes the established resident strain and $y$ the mutant invader (Metz et al., 1992). For this model the fitness, in terms of $b_{i j}, e_{i i}$ and $t_{i j}$ is given by

$$
s_{x}(y) \propto \sum_{i=1}^{n}\left[\left(b_{1 i}-e_{i i}\right)\left(\prod_{j=0}^{i-1} t_{\{j+1\} j}\right)\left(\prod_{j=i+1}^{n+1}\left(t_{\{j-1\} j}+e_{j j}\right)\right)\right] .
$$

(See Appendix B.1 for the derivation of Eq. (5).)

Each of the three criteria is claimed to have a specific effect on the shape of the invasion boundaries in a TIP with resultant evolutionary repercussions. Satisfying criterion A (appearing criterion) is necessary for the invasion boundaries to curve and/or separate (details depend on the other criteria), satisfying criterion $\mathrm{B}$ (characteristic criterion) is necessary for the invasion boundary $f_{1}$, stemming from $s_{x}(y)$, to curve and satisfying criterion $\mathrm{C}$ (density dependent rates criterion) is necessary for the invasion boundaries to separate. However whether an effect is possible or not can depend on the status of the other criteria (see Table 1). For example, to allow the possibility that invasion boundaries separate, and hence allow branching points and Garden of Eden points (ESSrepellors), it is actually necessary for both criteria A and $\mathrm{C}$ to be satisfied; $\mathrm{A}$ 
or $\mathrm{C}$ alone will not be enough.

We prove below that the criteria are necessary for the said effects by showing, for each criterion in turn, that, without it being satisfied, the corresponding effect on the invasion boundaries cannot occur. (In each case the status of the other two criteria is irrelevant.)

\subsection{Criterion A: The appearing criterion}

This criterion relates to where, in the matrix $\mathbf{M}$, the evolving parameters appear, and hence for which classes/species these parameters have a direct effect on the rate of change. For this criterion not to be satisfied requires that all the evolving parameters must be contained in a single row of $\mathbf{M}$; all the other rows of $\mathbf{M}$ must not be directly affected by changes in the evolving parameters. An equivalent explanation is that all the evolving parameters must be contained in a single equation describing the dynamics; all other equations must not be directly affected by changes in the evolving parameters.

For the case when the evolving species is described by a single equation (class) (i.e. $n=1$ ) - in a Lotka-Volterra set-up, where all evolving parameters only appear in a single class - it has been shown that not only are branching points and Garden of Eden points not possible (White and Bowers 2005) but the curvatures of the invasion boundaries at the evolutionary singularity are equal (Bowers et al. 2005). For example, for prey evolution in a predator-prey system (where the prey dynamics consist of only a single class), for the criterion to hold, the evolving parameters must appear in (i.e. directly affect) both the dynamics describing the prey and the dynamics descibing the predator. 
This can (usually) only occur when the evolving parameters affect the rate of predation and appear in both the dynamics of the prey and the dynamics of the predator. Hence although the predator is not evolving, its ability to predate as described by its dynamics is directly affected due to the evolution of the prey.

We now assume that the criterion does not hold, hence only a type I TIP should occur, and deduce the consequences.

First we write the fitness as $s_{x}(y)=s\left(y, X_{i}(x)\right)$ (fitness is determined by the mutant strain $y$ and the densities of the established resident and any nonevolving species). We note here that although the mutant strain is described by its two (evolving) traits, $y_{1}$ and $y_{2}$, these are linked by a trade-off, $y_{2}=f\left(y_{1}\right)$ say, and hence it is possible to reduce this to a single trait, $y_{1}$ say (for the working in this paper we do not require the trade-off to be shown explicitly). For convenience we abuse notation and drop the subscripts and write $y$ rather than $y_{1}$ (and $x$ rather than $x_{1}$ ). Differentiating this both with respect to the established resident and with respect to the mutant invader, and evaluating at the evolutionary singularity (Metz et al., 1996a; Geritz et al., 1998), gives

$$
\left.\frac{\partial^{2} s_{x}(y)}{\partial x \partial y}\right|_{x^{*}}=\left.\left.\sum_{i=1}^{m} \frac{\partial^{2} s}{\partial X_{i} \partial y}\right|_{x^{*}} \frac{d X_{i}}{d x}\right|_{x^{*}},
$$

where $\left.\left.\right|_{x^{*}} \Leftrightarrow\right|_{y=x=x^{*}}$. Bowers et al. (2005) show that if the mixed derivative of the fitness is zero at the evolutionary singularity then the two invasion boundaries will have equal curvatures at $x^{*}$ and hence will be superimposed (if approximated up to quadratic terms). We aim to show this is the case here by proving that the $d X_{i} /\left.d x\right|_{x^{*}}$ are all zero.

We begin with the observation that when the dynamics describing the evolving 
species are made up of more than one class (i.e. $n>1$ ), it follows from our assumption that the criterion does not hold that, the evolving parameters cannot affect the transition terms, $t_{i j}=t_{\{j+1\}}$, as these appear in two different rows of $\mathbf{M}$ and hence in the dynamics describing both class $j$ and class $j+1$ (or class $n$ and class 1 for $t_{1 n}$ ); hence only the $b_{i j}$ and $e_{i i}$ terms can depend on the evolving parameters.

Due to how we have set up the dynamics of our model we must now consider two distinct cases (the evolving parameters must appear somewhere in the dynamics of the evolving species):

\subsubsection{The evolving parameters only appear in the dynamics describing class 1}

In the present case, the evolving parameters can only appear in the reproduction terms, $b_{i j}=b_{1 j}$, or the mortality term related to class $1, e_{11}$; hence these are the only terms varying as the species evolves and hence the only functions of $x$ or $y$.

Focussing on the resident dynamics describing class 1, in Eq. (3), and differentiating with respect to $x$, taking into account the dependencies $X_{i}(x)$ and $\bar{b}_{i j}=B_{i j}\left(x, X_{1}(x), \ldots, X_{m}(x)\right)$, gives

$$
\begin{array}{r}
\sum_{j=1}^{m} \frac{\partial}{\partial X_{j}}\left(\sum_{i=1}^{n} \bar{b}_{1 i} X_{i}-\bar{e}_{11} X_{1}-\bar{t}_{21} X_{1}+\bar{t}_{1 n} X_{n}\right) \frac{d X_{j}}{d x} \\
+\sum_{i=1}^{n} \frac{\partial \bar{b}_{1 i}}{\partial x} X_{i}-\frac{\partial \bar{e}_{11}}{\partial x} X_{1}=0 .
\end{array}
$$

Our aim is to evaluate this at the evolutionary singularity. This requires the fitness gradient. 
Differentiating the fitness in Eq. (5) with respect to $y$, evaluating at the evolutionary singularity $x^{*}$ (Metz et al., 1996a; Geritz et al., 1998), gives

$$
\begin{aligned}
\left.\left.\frac{\partial s_{x}(y)}{\partial y}\right|_{x^{*}} \propto \sum_{i=1}^{n} \frac{\partial b_{1 i}}{\partial y}\right|_{x^{*}} & \left(\prod_{j=0}^{i-1} \bar{t}_{\{j+1\} j}\right)\left(\prod_{j=i+1}^{n+1}\left(\bar{t}_{\{j+1\} j}+\bar{e}_{j j}\right)\right) \\
& -\left.\frac{\partial e_{11}}{\partial y}\right|_{x^{*}}\left(t_{10}\right)\left(\prod_{j=2}^{n+1}\left(\bar{t}_{\{j+1\} j}+\bar{e}_{j j}\right)\right) .
\end{aligned}
$$

Returning to the resident dynamics, in Eq. (3), the dynamics describing classes 2 to $n$ yield the set of equations

$$
X_{i}=\frac{\bar{t}_{i\{i-1\}}}{\left(\bar{t}_{\{i+1\} i}+\bar{e}_{i i}\right)} X_{i-1} \text { for } i=2, . ., n .
$$

Solving these gives

$$
X_{i}=\frac{\prod_{j=1}^{i-1} \bar{t}_{\{j+1\} j}}{\prod_{j=2}^{i}\left(\bar{t}_{\{j+1\} j}+\bar{e}_{j j}\right)} X_{1}, \quad i=2, \ldots, n .
$$

Using this, we can re-write the derivative of $s_{x}(y)$, in Eq. (8), as

$$
\left.\frac{\partial s_{x}(y)}{\partial y}\right|_{x^{*}} \propto\left(\left.\sum_{i=1}^{n} \frac{\partial b_{1 i}}{\partial y}\right|_{x^{*}} \frac{X_{i}^{*}}{X_{1}^{*}}-\left.\frac{\partial e_{11}}{\partial y}\right|_{x^{*}}\right) \prod_{j=2}^{n}\left(\bar{t}_{\{j+1\} j}+\bar{e}_{j j}\right) .
$$

As this is zero at the evolutionary singularity, $x^{*}$, and since $t_{\{i+1\} i}+e_{i i}>0$ for all $i$ (see the discussion near Eq. (B.5)), we find that

$$
\left.\sum_{i=1}^{n} X_{i}^{*} \frac{\partial b_{1 i}}{\partial y}\right|_{x^{*}}=\left.X_{1}^{*} \frac{\partial e_{11}}{\partial y}\right|_{x^{*}}
$$

Eq. (12) allows us to simplify Eq. (7) at the singularity to give

$$
\left.A_{11} \frac{d X_{1}}{d x}\right|_{x^{*}}+\left.A_{12} \frac{d X_{2}}{d x}\right|_{x^{*}}+\ldots+\left.A_{1 m} \frac{d X_{m}}{d x}\right|_{x^{*}}=0
$$

where $A_{i j}$ are functions of $b_{i j}, e_{i i}$ and $t_{i j}$, and their derivatives with respect to the densities $X_{j}$, evaluated at the singularity. 
If we had written Eq. (1) in the appropriate form

$$
\frac{d X_{i}}{d t}=F_{i}\left(x, X_{j}(x)\right) \text { for } i=1, \ldots, m
$$

then we should have found $A_{i j}=\partial F_{i} /\left.\partial X_{j}\right|_{x^{*}}$. Similarly to Eq. (13), since the $F_{i}$ have no explicit $x$ dependence for $i>2$, we have

$$
\sum_{j} A_{i j} \frac{d X_{j}}{d x}=0 \quad \text { for } i>2
$$

Thus Eq. (13) and Eq. (15) yield

$$
\mathbf{A}\left(\left.\frac{d X_{j}}{d x}\right|_{x^{*}}\right)^{T}=0
$$

where $\mathbf{A}$ is the Jacobian of the $F_{i}$ with respect to the $X_{j}$ at $x^{*}$. Since we assume $x^{*}$ is point stable in the population dynamics, $\mathbf{A}$ is non-singular and So

$$
\left.\frac{d X_{i}}{d x}\right|_{x^{*}}=0 \quad \text { for } i=1, \ldots, m .
$$

Hence, returning to Eq. (6), we see that the mixed derivative of $s_{x}(y)$ is zero at the singularity and therefore the invasion boundaries must be superimposed. We briefly note that the result in Eq. (17) shows that at the evolutionary singularity, the population density of all classes attains an extremum. This suggests the possiblility of optimisation in this model, which again excludes the possibility of co-existence of strains not only locally (which leads to branching ponts not being possible) but also globally.

The linearity of the invasion boundaries comes about by the fact that the fitness, in Eq. (5), is linear in terms of $b_{1 i}$ and $e_{11}$ (the terms containing the evolving parameters). Combining this with an assumption we make concerning 
the evolving parameters appearing linearly in the dynamics, and hence in $b_{1 i}$ and $e_{11}$, then the invasion boundary stemming from $s_{x}(y)$ will be linear in terms of the evolving parameters. Further, as the invasion boundaries are superimposed, the second invasion boundary must also be straight giving a type I TIP.

3.1.2 The evolving parameters only appear in the dynamics describing class $k$, where $k \neq 1$

In the case where the evolving parameters only appear in the dynamics describing a single class which is not class 1 (i.e. a class in which there are no individuals entering through birth), they can only exist in the mortality term, $e_{k k}($ where $k \neq 1)$ - this appears in one class only. Following the proof through as in the case above (for $k=1$ ), see Appendix B.2 for details, again yields the result that the two invasion boundaries are superimposed and linear. Thus we have established the results in the first row of Table 1, such that not satisfying criterion A leads to linear and superimposed invasion boundaries with the evolutionary consequences - that acceleratingly costly trade-offs produce evolutionary attractors and deceleratingly costly trade-offs lead to repellors.

\subsection{Criterion $B$ : the characteristic criterion}

This criterion again relates to the evolving parameters but now is concerned with whether they are characteristics of more than one class and hence appear in more than one column of $\mathbf{M}^{(n)}$. If this criterion is satisfied, then - provided criterion A (appearing) is too - it is possible for the invasion boundary $f_{1}$ stemming from $s_{x}(y)=0$ - to curve and give a type II or a type IV TIP (see 
Fig. 1). In contrast, if this criterion is not satisfied then it is only possible to get a type I or a type III TIP (see Fig. 1). We again assume that the criterion does not hold and deduce the consequences.

Given that the evolving parameters are characteristics of the same class, and hence only appear in a single column of $\mathbf{M}$, then the rates, $b_{i j}, e_{i j}$ and $t_{i j}$, affected by these parameters will all have the same $j$. Looking back to the form of the fitness in Eq. (5), we see that it is linear in terms of $b_{i j}, e_{i j}$ and $t_{i j}$ for a given $j$. In addition, taking into account our assumption earlier regarding the linearity of (the evolving) parameters, it follows that these appear linearly in the fitness. Hence, the invasion boundary stemming from $s_{x}(y)$ (the $f_{1}$ boundary) will be linear in terms of the evolving parameters.

Satisfying criterion $\mathrm{B}$ is necessary in order for the $f_{1}$ invasion boundary to curve; however satisfying it is not sufficient to ensure the boundary curves. For example, if the evolving parameters affect $b_{12}$ and $e_{11}$, and hence are characteristics of classes 1 and 2, these rates will still appear linearly in the fitness, in Eq. (5), and hence the invasion boundary $f_{1}$ will be straight.

\subsection{Criterion C: density dependent rates criterion}

The third criterion is concerned with how all the entries $M_{i j}^{(n)}$, and hence $b_{i j}$, $e_{i i}$ and $t_{i j}$, depend upon the population densities of the resident strain (and any non-evolving species). For criterion $\mathrm{C}$ to be satisfied requires there to be at least two density dependent rates in the dynamics. These two (or more) rates can occur in the same $b_{i j}, e_{i i}$ or $t_{i j}$ term; there is no requirement for two different terms to be density dependent. In addition these (at least) two 
density dependent rates must not depend upon the same densities in the same manner.

If the criterion is satisfied, then - provided criterion A (appearing) is too - the invasion boundaries can separate, producing either a type III or type IV TIP (see Fig. 1), depending upon whether the remaining criterion (B) is satisfied. If criterion $\mathrm{C}$ is not satisfied, then the boundaries cannot separate. We again use the result (Bowers et al., 2005) that the boundaries having equal curvature at the tip of the singular TIP (i.e. being locally superimposed) is equivalent to the mixed derivative of the fitness being zero at the evolutionary singularity.

The resultant effect on the fitness of not satisfying criterion $\mathrm{C}$ is that it is only a function of the evolving parameters, which are dependent on $y$, and of one function $X(x)$ (a single $X_{i}$ or some combination of these densities), and hence will be of the form $s_{x}(y)=s(y, X(x))$. Thus $\partial s_{x}(y) / \partial x=(\partial s / \partial X)(d X / d x)$ and since this derivative is zero at the singularity (Metz et al., 1996a; Geritz et al., 1998) $d X /\left.d x\right|_{x^{*}}=0$. Since $\partial^{2} s_{x}(y) / \partial y \partial x=\left(\partial^{2} s / \partial y \partial X\right)(d X / d x)$, this mixed derivative is zero at the singularity.

The idea that if the fitness is only a function of a single density, and the traits of the mutant strain, then branching points and Garden of Eden points (ESS-repellors) are impossible, is not a recent one (for example, see Metz et al., 1996b; Heino et al., 1998; Kisdi, 1998; Rueffler et al., 2006). A fitness of this form has been called one dimensional feedback environment, frequency independent and 'trivial' frequency dependence (see previous references). The benefit of taking this idea one step back (from the fitness to the dynamics) is that whether separation of boundaries is possible or not can be determined without carrying out the often complex calculations in order to find the fitness 
function.

\section{Relaxing the technical assumptions}

In the above analysis a number of 'technical' assumptions were made, in particular: that all individuals enter into class 1 and then may move through the classes sequentially, eventually possibly returning to class 1 from the last class. However in many ecological systems these are not the case. By relaxing the assumptions in turn, we will test whether each of our three criteria still hold.

\subsection{Individuals born into different classes}

We begin by holding to the assumption regarding individuals moving through the classes in order. However, we relax the assumption concerning into which class individuals are born. Previously we had all new individuals entering into class 1 . However, if we consider SIR systems, vertical transmission or natural immunity can lead to offspring directly entering the infected or recovered class, respectively. Taking this into account, the dynamics (of our evolving species) now take the form

$$
\frac{d X_{i}}{d t}=\sum_{j=1}^{n} \bar{b}_{i j} X_{j}-\bar{e}_{i i} X_{i}-\bar{t}_{\{i+1\} i} X_{i}+\bar{t}_{i\{i-1\}} X_{i-1}, \quad \text { for } i=1, \ldots, n
$$

where we replace $+\bar{t}_{10} X_{0}$, for $j=1$, and $-\bar{t}_{\{n+1\} n} X_{n}$, for $j=n$, with $\pm \bar{t}_{1 n} X_{n}$, respectively, so that 'cycling' is explicitly included. For simplifying purposes we take $n=2$, i.e. the evolving species only consists of two classes. Fig. 3 shows the life cycle graph for this model. If we introduce a mutation in the evolving species, the dynamics of the rare invader will take the form 


$$
\begin{aligned}
& \frac{d Y_{1}}{d t}=b_{11} Y_{1}+b_{12} Y_{2}-e_{11} Y_{1}-t_{21} Y_{1}+t_{12} Y_{2}, \\
& \frac{d Y_{2}}{d t}=b_{21} Y_{1}+b_{22} Y_{2}-e_{22} Y_{2}-t_{12} Y_{2}+t_{21} Y_{1} .
\end{aligned}
$$

We can show that although the current assumption has been relaxed, the three criteria still hold, in that satisfying them allows the relevant TIP type, and resultant evolutionary outcomes, to be possible (see Appendix C.1 for details). Although this is limited to $n=2$, we expect that the results will apply more generally.

\subsection{Individuals do not move through classes in order}

Previously, we made an assumption that individuals move through the classes sequentially, perhaps returning to the first class from the last. However in a number of ecological systems this is not always the case. For example, in an SIR model, individuals can be born into the susceptible class and from there they can be infected, moving into the infected class, or they can be vaccinated, moving straight to the immune (removed) class avoiding the infected class completely. For this reason, we now take a model where we again assume that all individuals are born into class 1; however, we allow movement between any class (i.e. they begin in class 1 and from there they can move to any of the other $n-1$ classes, from which they can again move to any of the $n-1$ classes).

Here the dynamics (of our evolving species) take the form 


$$
\begin{aligned}
& \frac{d X_{1}}{d t}=\sum_{j=1}^{n} \bar{b}_{1 j} X_{j}-\bar{e}_{11} X_{1}+\sum_{j=1}^{n}\left(\bar{t}_{1 j} X_{j}-\bar{t}_{j 1} X_{1}\right), \\
& \frac{d X_{i}}{d t}=-\bar{e}_{i i} X_{i}+\sum_{j=1}^{n}\left(\bar{t}_{i j} X_{j}-\bar{t}_{j i} X_{i}\right) \quad \text { for } i=2, \ldots, n .
\end{aligned}
$$

For simplicity, we take a system where the evolving species consists of 3 classes as this is the smallest number which creates differences in the dynamics from the previous models. The life cycle graph is shown in Fig. 4. In this model, a (rare) invader strain will have dynamics

$$
\begin{aligned}
& \frac{d Y_{1}}{d t}=\sum_{i=1}^{3} b_{1 i} Y_{i}-e_{11} Y_{1}+\sum_{i=1}^{3}\left(t_{1 i} Y_{i}-t_{i 1} Y_{1}\right) \\
& \frac{d Y_{2}}{d t}=-e_{22} Y_{2}+\sum_{i=1}^{3}\left(t_{2 i} Y_{i}-t_{i 2} Y_{2}\right) \\
& \frac{d Y_{3}}{d t}=-e_{33} Y_{3}+\sum_{i=1}^{3}\left(t_{3 i} Y_{i}-t_{i 3} Y_{3}\right)
\end{aligned}
$$

We can again show that although the assumption has been relaxed the three criteria still hold, giving the possible TIPs stated in Table 1 and respective evolutionary outcomes (see Appendix C.2 for details).

\section{Evolving parameters: non-linearities and coordinate change}

In many systems (for example, Bowers et al., 2003; White and Bowers, 2005) evolving parameters which are used as coordinates in presenting our TIPs may be identified on biological grounds - per capita low density birth rates or recovery rates from infection - and then observed to appear linearly in the dynamics. Despite this there are two interrelated reasons for investigating the effects on our criteria of parameters which enter $\mathbf{M}$ non-linearly. First, in more complex settings, parameters of direct biological importance may enter nonlinearly - an example of this appears in studies of the evolution of predator 
handling time using a Holling Type II functional response (Holling, 1969; Kisdi and Liu, 2006; Hoyle and Bowers, 2007; Geritz et al., 2007). Second, it may be inappropriate to afford certain parameters - in particular those entering linearly - a privileged position on the basis of a notion of 'direct biological importance'. For example, why should a recovery rate be stressed rather than the corresponding duration of infection? This perspective stresses those aspects of our analysis which are invariant under appropriate smooth coordinate change in the space of evolving parameters. Invariably, coordinate change will produce 'new parameters' which enter the dynamics non-linearly; the issues are essentially the same.

In the models we have studied so far - with the evolving parameters (adopted as coordinates) appearing linearly - not satisfying criterion A results in the invasion boundaries being both linear and superimposed. If we remove the assumption that the evolving parameters appear linearly, we can still use the method of section 3.1 to show that, when criterion A is not satisfied, the invasion boundaries are superimposed (the argument makes no assumption about parameter linearity). However, the argument showing that when criterion $\mathrm{A}$ is not satisfied, the invasion boundaries are linear does depend on the parameter linearity; this property is not generic under parameter choice. Generically, not satisfying criterion A gives a type II TIP with type I as a degenerate case.

In the models we have studied so far - with the evolving parameters appearing linearly - not satisfying criterion B results in the invasion boundary $f_{1}$ being linear. If we remove the assumption that the evolving parameters appear linearly, this conclusion no longer applies. (The fitness is no longer linear in the evolving parameters.) Hence generically, this criterion has no power. 
In the same context, criterion $\mathrm{C}$ still applies. The argument in section 3.3 is unaffected by parameter non-linearity. Thus generically not satisfying criterion C implies superimposed boundaries which again means a type II TIP with type I as a degenerate case.

The final conclusion that can be drawn is that both criteria $\mathrm{A}$ and $\mathrm{C}$ are necessary for separated boundaries which result in a type IV TIP, the degenerate case now being type III.

However, despite the criteria only being partially valid in the above cases, we emphasise the significance of the three criteria and the resultant classification of the four TIP types in the important cases where the parameters are linear.

\section{Discussion}

Trade-off and invasion plots (TIPs) (Bowers et al., 2005) were developed as a graphical alternative, which keeps the trade-off explicit, to the traditional approach to adaptive dynamics (Metz et al., 1996a; Geritz et al., 1998). The key determining factor of which evolutionary outcome occurs is the respective curvatures of the trade-off and the invasion boundaries. Hoyle et al. (2008) introduced four fundamental types of TIP (Fig. 1), each with immediate consequences for the possible evolutionary behaviour. In addition, these authors introduced three criteria (based on various aspects of continuous time models) and claimed, on the basis of a few model calculations, that their occurence in various combinations leads to a classification of possible TIPs. These three criteria were the appearing criterion (A), the characteristic criterion (B) and the density dependent rates criterion $(\mathrm{C})$. The combinations of these crite- 
ria and the respective TIPs produced are shown in Table 1 and Fig. 1. We have presented proofs of these claims for a relatively general continuous time set-up. Our analysis initially depended on various assumptions: $(i)$ that all individuals enter class 1 and $(i i)$ then move through the classes sequentially, possibly returning to class 1 from the final class, and (iii) that the evolving parameters appear linearly in the dynamics.

A key feature shown however is that although the criteria are necessary to gain each type of TIP, they are not sufficient to guarantee that type. For example, suppose we return to the dynamics in Eq. (3) and take a situation where the evolving parameters are contained in the terms $b_{1 i}$ and $e_{\{i-1\}\{i-1\}}$ (a trade-off between the birth rate from individuals in class $i$ and the death rate of individuals in class $i-1)$. Here, the evolving parameters appear in (directly affect) the dynamics describing more than one class (satisfying criterion A) and are characteristics of different classes (satisfying criterion B), therefore we might expect to gain a type II (i.e. two curved, superimposed boundaries) or type IV TIP (i.e. two curved, separated boundaries). However, because $b_{1 i}$ and $e_{\{i-1\}\{i-1\}}$ appear in the fitness, $s_{x}(y)$ in Eq. (5), linearly (i.e. $b_{i}$ does not multiply $\left.e_{\{i-1\}\{i-1\}}\right)$, the invasion boundary stemming from $s_{x}(y)$ will be linear in terms of evolving parameters (as these appear linearly in $b_{1 i}$ and $\left.e_{\{i-1\}\{i-1\}}\right)$. Therefore the TIP will either have two straight, superimposed invasion boundaries (type I) or one straight and one curved boundary (type III). Hence being characteristic of different classes is necessary for the boundary stemming from $s_{x}(y)$ to curve, but it is not sufficient for this to be the case.

In order to expand the spectrum of models where our criteria (might) hold, for example to SIR systems with vertical transmission or vaccination, we relaxed 
each of the main three assumptions in turn. We showed that if we relaxed the assumptions regarding into which classes new individuals enter and the order in which individuals move through the classes, then the three criteria still applied for cases when the evolving species were made up of 2 and 3 classes respectively. We expect that the criteria will hold for cases when the evolving species is made up of any number of classes.

When we relaxed the final assumption, that the evolving parameters enter the dynamics linearly, to allow for example for handling times in a Holling Type II functional response in predator-prey systems and smooth coordinate changes, we found that although criterion C still holds, criteria A and B fail. Concerning criterion A however, if the evolving parameters only appear in a single class, then the part of the criterion stating that the invasion boundaries are superimposed holds; however the part stating that the boundaries are straight fails. Therefore, where previously not satisfying criterion A gave a type I TIP only, if the evolving parameters appear non-linearly then this now gives either a type I or type II TIP. The only way to get a type III or type IV TIP is if both criteria A and C are satisfied; if either (or neither) are satisfied, then only type I or type II TIPs are possible. Here criterion B does not play a part in which type of TIP is produced.

Prior to this work the link between TIPs and evolutionary behaviour had already been obtained by adding trade-off curves to a TIP (see Fig. 5 for an example) as discussed fully in Bowers et al. (2005). We now link the occurence or otherwise of criteria $\mathrm{A}, \mathrm{B}$ and $\mathrm{C}$ directly to evolutionary behaviour in more detail using the TIP as a link. This works for a relatively broad class of ecological systems described near Eq. (3), made broader by our later extensions. Thus for systems in which the evolving parameters directly affect only one 
class/species (not A - e.g. $a_{x}$ and $q_{x}$ in Eq. (2)), or those in which the evolving parameters do affect more than one class/species, the evolving parameters are characteristics of only one class, and the rates are dependent on only one density or combination of densities (which are therefore A, not B, not C - e.g. $a_{x}$ and $\beta_{x}$ in Eq. (2) with $q_{x}=0$ ), we have a type I TIP and hence acceleratingly costly trade-offs lead to evolutionary attractors and deceleratingly costly trade-offs lead to evolutionary repellors (as seen in Fig. 5 - Type I). Systems in which both the parameter criteria are satisfied but the rates remain dependent on only one density or combination of densities (which are therefore A, B, not $\mathrm{C}$ - e.g. $a_{x}$ and $\alpha_{x}$ in Eq. (2) with $q_{x}=0$ ) may be type II when, if the superimposed invasion boundaries curve in the manner in Fig. 5 , strongly deceleratingly costly trade-offs lead to repellors, and weakly deceleratingly costly and acceleratingly costly trade-offs lead to attractors (and correspondingly). Systems in which the first (appearing) parameter criterion and the rates criterion are satisfied but the evolving parameters remain characteristic of only one class (A, not B, C - e.g. $a_{x}$ and $\beta_{x}$ Eq. (2)) may be Type III when, if the $f_{2}$ boundary curves as in Fig. 5, strongly decelerating costly trade-offs lead to repellors, acceleratingly costly trade-offs lead to attractors but weakly deceleratingly costly trade-offs now lead to branching points. Finally, systems in which all the criteria are satisfied (A, B, C - e.g. $a_{x}$ and $\alpha_{x}$ in Eq. (2)) may be type IV when, if the configuration is as in Fig. 5, in addition to the type III results, weakly acceleratingly costly trade-offs may also yield branching points. Although branching points have been shown for certain regions between the two invasion boundaries, in Fig. 5, Garden of Eden points (ESS-repellors) may occur instead. Which of these, branching points or Garden of Eden points, occur for relevant shaped trade-offs depends upon the relative curvatures of the invasion boundaries at the evolutionary singularity 
(i.e. whether the curvature of $f_{1}$ is greater than that of $f_{2}$, or vice-versa, near $\left.x^{*}\right)$ and the signs of the fitness funtions on either side of the invasion boundaries (e.g. whether $s_{x^{*}}(y)>0$ above or below the $f_{1}$ invasion boundary). The possible evolutionary outcomes for the various shapes of trade-off for each possible configuration are shown in Table A.1.

Since the occurence of branching points is linked to dimorphism and possibly speciation (Metz et al., 1996a; Geritz et al., 1998; Doebeli and Dieckmann, 2000), it is intriguing to observe that necessary conditions for these are that the evolving parameters directly affect not only one class/species (A) and that the rates are not dependent on only one density or combination of densities (C). Whether these apply or not can be obtained directly from the model without the need for further calculation. Given these and weakly deceleratingly costly trade-offs (type III, A, not B, C) or appropriate trade-offs of intermediate strength (type IV, A, B, C) branching points are possible for the class of system studied here; they are not otherwise.

\section{Acknowledgements}

We wish to thank the reviewers for very useful comments on earlier drafts of this article; we are also grateful to Dr. A. White and Prof. M. Boots for many helpful conversations. 


\section{References}

Anderson, R.M., May, R.M.,1979. Population biology of infectious diseases: Part I. Nature 280, 361-367.

Boots, M., Haraguchi, Y., 1999. The evolution of costly resistance in hostparasite systems. Am. Nat. 153, 359-370.

Boots, M., Bowers, R.G., 2004. The evolution of resistance through costly acquired immunity. Proc. Roy. Soc. Lond. B 271, 715-723.

Bowers, R.G., White, A., 2002. The adaptive dynamics of Lotka-Volterra systems with trade-offs. Math. Biosci. 175, 67-81.

Bowers, R.G., White, A., Boots, M., Geritz, S.A.H., Kisdi, E., 2003. Evolutionary branching/speciation: contrasting results from systems with explicit or emergent carrying capacities. Evol. Ecol. Res. 5, 883-891.

Bowers, R.G., Hoyle, A., White, A., Boots M., 2005. The geometric theory of adaptive evolution: trade-off and invasion plots. J. Theor. Biol. 233, 363-377.

Dercole, F., Rinaldi, S., 2002. Evolution of cannibalistic traits: scenarios derived from adaptive dynamics. Theor. Pop. Biol. 62, 365-374.

Diekmann, O., Heesterbeek, J.A.P., 2000. Mathematical epidemiology of infectious diseases: model building, analysis and interpretation. Wiley Series in Mathematical and Computationaly Biology, Wiley, Chichester.

De Mazancourt, C., Dieckmann, U., 2004. Trade-off geometries and frequencydependent selectio. Am. Nat. 164, 765-778.

Doebeli, M., Dieckmann, U., 2000. Evolutionary branching and sympatric speciation caused by different types of ecological interactions. Am. Nat. 156, $77-101$.

Geritz, S.A.H., Kisdi, E., Meszena, G., Metz, J.A.J., 1998. Evolutionary singular strategies and the adaptive growth and branching of the evolutionary tree. J. Evol. Ecol. 12, 35-57.

Geritz, S.A.H., Kisdi, E., Yan, P., 2007. Evolutionary branching and long-term 
coexistence of cycling predators: Critical function analysis. Theor. Pop. Biol. $71,424-435$.

Heino, M., Metz, J.A.J, Kaitala, V., 1998. The enigma of frequency-dependent selection. Trends Ecol. Evol. 13, 367-370.

Holling, C.S., 1959. The components of predation as revealed by a study of small-mammal predation of the European pine sawfly. Canadian Entomologist $91,293-320$.

Hoyle, A., Bowers, R.G., 2007. When is evolutionary branching in predatorprey systems possible with an explicit carrying capacity. Math. Biosci. 210, $1-16$.

Hoyle, A., Bowers, R.G., White, A., Boots, M., 2008. The influence of trade-off shape on evolutionary behaviour in classical ecological scenarios. J. Theor. Biol. 259, 498-511.

Kisdi, E., 1998. Frequency dependence versus optimization. Trends Ecol. Evol. $13,508$.

Kisdi, E., Liu, S., 2006. Evolution of handling time can destroy the coexistence of cycling predators. J. Evol. Ecol. 19, 49-58.

Levins, R., 1962. Theory of fitness in heterogeneous environment. 1. the fitness set and the adaptive function. Am. Nat. 96, 361-373.

Levins, R., 1968. Evolution in changing environments. Princeton University Press, Princeton.

Loeuille, N., Loreau, M., 2004. Nutrient enrichment and food chains: can evolution buffer top-down control? Theor. Pop. Biol. 65, 285-298.

Metz, J.A.J., Nisbet, R.M., Geritz, S.A.H., 1992. How should we define 'fitness' for general ecological scenarios? Trends Ecol. Evol. 7, 198-202.

Metz, J.A.J., Geritz, S.A.H., Meszena, G., Jacobs, F.J.A., Van Heerwaarden, J.S., 1996a. Adaptive dynamics: A geometric study of the consequences of nearly faithful reproduction. In: S.J. Van Strien, S.M. Verduyn Lunel, (Ed.). 
Stochastic and Spatial Structures of Dynamical Systems, Elsevier, NorthHolland, Amsterdam, pp. 183-231.

Metz, J.A.J., Mylius, S.D., Diekmann, O., 1996b. When does evolution optimise? On the relation between types of density dependence and evolutionarily stable life history parameters. IIASA working paper WP-96-004, available at http://www.iiasa.ac.at/Research/ADN/Series.html.

Reade, B., Bowers, R.G., Begon, M., Gaskell, R., 1998. A model of disease and vaccination for infections with acute and chronic phases. J. Theor. Biol. 190, 355-367.

Roff, D.A., 2002. Life history evolution. Sinauer, Sunderland, MA.

Rueffler, C., Van Dooren, T.J.M., Metz, J.A.J., 2004. Adaptive walks on changing landscapes: Levin's approach. Theor. Pop. Biol. 65, 165-178.

Rueffler, C., Van Dooren, T.J.M., Metz, J.A.J., 2006. The evolution of resource specialization through frequency-dependent and frequency-independent mechanisms. Am. Nat. 167, 81-93.

Stearns, S.C., 1992. The evolution of life histories. Oxford University Press, Oxford.

White, A., Bowers, R.G., 2005. Adaptive dynamics of Lotka-Volterra systems with trade-offs: the role of intraspecific parameter dependence in branching. Math BioSci. 197, 101-117. 


\section{A Trade-off and invasion plots (TIPs)}

A detailed description of the use of trade-off and invasion plots (TIPs) to determine evolutionary behaviour has been given elsewhere (Bowers et al., 2005). Here we will give a brief outline of TIPs and present some of the results/conditions for determining the evolutionary behaviour of a system.

Trade-off and invasion plots are a geometrical approach that makes the role that different trade-off shapes play easy to visualise. A TIP is a plot between two (competing) strains of a species, labelled $x$ and $y$ say. One of these, $x$, is taken to be fixed while the second, $y$, is allowed to vary. The axes of a TIP are the two evolving parameters (or traits) of the $y$ strain, $y_{1}$ and $y_{2}$ (only two parameters are taken to vary). The co-ordinates $x_{1}$ and $x_{2}$ of the fixed strain $x$ define the corner or tip of a TIP. Examples of TIPs (including the evolutionary outcomes for each region) can be seen in Fig. 5.

Two of the three curves on a TIP are the invasion boundaries, denoted as $f_{1}$ and $f_{2}$. These curves denote where $s_{x}(y)=0$ and $s_{y}(x)=0$ respectively, and hence into regions where the varying strain $y$ can and cannot invade the fixed strain $x$ (either side of $f_{1}$ - where $s_{x}(y)>0$ and $s_{x}(y)<0$ respectively) and where the fixed strain $x$ can and cannot invade the varying strain $y$ (either side of $f_{2}$ - where $s_{y}(x)>0$ and $s_{y}(x)<0$ respectively). Both of these invasion boundaries pass through the tip of a TIP (where $y=x$ ) at which they are tangential. The third curve on a TIP is the trade-off curve, denoted as $f$; this links the two evolving parameters of each strain. This curve also passes through the tip of a TIP, but not usually tangentially to the invasion boundaries. Importantly, as all the feasible pairs of traits (and hence strains) lie on his 
curve, the side of the invasion boundaries in which the trade-off enters a TIP determines whether each strain can invade the other (when initially rare).

For certain TIPs corresponding to particular values $x^{*}$ of $x$, the trade-off curve can become tangential to the invasion boundaries at the tip of a TIP (i.e. where $y=x=x^{*}$ ); these values of $x$ are evolutionary singularities, with the corresponding TIPs being singular TIPs (Fig. 5). It is from these singular TIPs that the evolutionary behaviour of a system is determined. The invasion boundaries (and their mean curvature) separate the singular TIP into regions, each with their own respective evolutionary behaviour. (If a singular point does not exist, then invadability will prefer either always higher or always lower values of $x$. If more than one singular point exists then a separate TIP must be considered at each singular point.) Due to the coincidence and mutual tangential property of the three curves at the tip of a singular TIP, the region in which the trade-off curve enters (and hence the evolutionary behaviour) is determined solely by the curvatures of the three curves; or more specifically, the curvature of the trade-off in relation to those of the invasion boundaries at the evolutionary singularity (as in standard theory mutations are assumed to be small). The two significant relations are between the trade-off and $f_{1}$ for evolutionary stability ESS and between the trade-off and the mean curvature of both $f_{1}$ and $f_{2}$ for convergent stability CS. These can be written

$$
\begin{aligned}
\mathrm{ESS} & \Leftrightarrow \lambda_{1} f^{\prime \prime}\left(x^{*}\right)<\left.\lambda_{1} \frac{\partial^{2} f_{1}}{\partial y^{2}}\right|_{x^{*}}, \\
\mathrm{CS} & \Leftrightarrow \lambda_{1} f^{\prime \prime}\left(x^{*}\right)<\frac{\lambda_{1}}{2}\left(\left.\frac{\partial^{2} f_{1}}{\partial y^{2}}\right|_{x^{*}}+\left.\frac{\partial^{2} f_{2}}{\partial y^{2}}\right|_{x^{*}}\right) .
\end{aligned}
$$

where $\lambda_{1}=\operatorname{sign}\left(s_{x^{*}}(y)\right)$ just above the invasion boundary $f_{1}$. Here $\lambda_{1}$ concerns how the fitness varies as we move vertically up a TIP (i.e. as we vary the 
parameter on the vertical axis). Combinations of these properties allow the evolutionary behaviour of the system to be determined. The possible types of singularity are evolutionary attractors or CSS (continuously stable strategy) (ESS and CS), evolutionary branching point (CS but not an ESS), 'Garden of Eden' point or ESS-repellor (ESS but not CS) and evolutionary repellor (neither ESS nor CS). The shapes of trade-off which lead to each of these are given in Table A.1. These conditions for ES and CS remain invariant under smooth changes of coordinates; hence if a particular evolutionary outcome occurs in one coordinate space, then it will occur in all. Examples of how these appear on a singular TIP are given in Fig. 5 for each of the four fundamental types of TIP.

\section{B Including assumptions regarding the birth and movement of in- dividuals}

\section{B.1 Derivation of the fitness function}

The fitness is defined as being the per capita growth rate of a rare mutant invader and is commonly denoted as $s_{x}(y)$, where $x$ denotes the established resident strain and $y$ the mutant invader (Metz et al., 1992). This fitness, or a sign equivalent version of it, can be found in a number of ways. A traditional method is to use $r$, the maximum eigenvalue of the invasion Jacobian (Metz et al., 1996a; Geritz et al., 1998). An alternative, which is sign equivalent, is to use $R_{0}-1$, where $R_{0}$ is the maximum eigenvalue of the next generation matrix. (Diekmann and Heesterbeek (2000) describe this equivalence in an epidemiological context.) Taking $\mathbf{G}$ as the transition matrix whose elements 
are the net rates of increase in individuals of class $i$ per individual of class $j$ (excluding reproduction terms) (Reade et al., 1998; Diekmann and Heesterbeek, 2000), the average times $T_{i j}$, which an individual born in class $j$ spends in class $i$, are identified as the elements of $-\mathbf{G}^{-1}$. Hence the next generation matrix is $-\mathbf{b}^{T} \mathbf{G}^{-1}$, where the elements of $\mathbf{b}$ are the per capita reproduction rates.

An assumption we make initially is that all individuals are born into a single class, this being class 1 . In this case all but the first row of $\mathbf{b}$ are null. Therefore the next generation matrix has a single non-zero eigenvalue $R_{0}$ and the fitness is

$$
R_{0}-1=\sum_{i=1}^{n} b_{1 i} T_{i 1}-1=\sum_{i=1}^{n}\left(b_{1 i}-e_{i i}\right) T_{i 1}
$$

The second equality here can be established formally as follows: The columns of $\mathbf{G}$ sum to the $-e_{i i}$ (since the diagonal elements are $-\left(e_{i i}+\sum_{j} t_{i j}\right)$ ) and hence $\operatorname{det}(\mathbf{G})=-\sum_{i} e_{i i} C_{1 i}$, where the $C_{i j}$ are cofactors. Thus

$$
\sum_{i=1}^{n} e_{i i} T_{i 1}=-\frac{1}{|\mathbf{G}|} \sum_{i=1}^{n} e_{i i} C_{1 i}=1
$$

as required. Thus, from Eq. (B.1), in the present form we can write down a sign equivalent fitness satisfiying

$$
s_{x}(y) \propto \sum_{i=1}^{n} \rho_{i} T_{i}
$$

Here we drop the second subscript and note that the growth rate terms, $\rho_{i}$, simply take the form $b_{1 i}-e_{i i}$, i.e. the difference between the reproduction terms and mortality terms corresponding to individuals in class $i$. We highlight here that these growth rates do not involve the transition terms, $t_{i j}$, as these are the 
rate at which individuals move from one class to the next and hence remain in the system without affecting the total population. For cases where the evolving species is made up of a single class (i.e. $n=1$ in the dynamics above), the only class is $Y_{1}$ which will have the dynamics $\left(b_{11}-e_{11}\right) Y_{1}$; hence the fitness will simply be the limit as $Y_{1} \rightarrow 0$ of $b_{11}-e_{11}$, i.e. of the difference between the reproduction and mortality terms.

Returning to our calculations for $T_{i}$, initially, an individual is born into class 1 and moves through the classes sequentially. Hence as it moves through the classes from 1 to $n$, the equations giving the time spent in each class, in terms of our mortality and transition terms are

$$
1=\sum_{i=1}^{k} e_{i i} T_{i}+t_{\{k+1\} k} T_{k} \text { for } k=1, \ldots, n,
$$

where here we have assumed that $t_{\{n+1\} n}=0$ so that individuals cannot return to class 1 from class $n$. Eqs. (B.4) can be supported phenomenologically since they equate to unity the probability of leaving (by mortality or transition to the next class) cumulatively to the end of each succesive class. They can also be established formally from $-\mathbf{G T}=\mathbf{I}$ in the case of sequential movement through the classes with no cycling.

The solution of Eq. (B.4), representing the average time spent in class $i$, is given by

$$
T_{i}=\frac{\prod_{j=0}^{i-1}\left(t_{\{j+1\} j}\right)}{\prod_{j=1}^{i}\left(t_{\{j+1\} j}+e_{j j}\right)} \quad \text { where } t_{10}=1
$$

Here we assume $t_{\{i+1\} i}+e_{i i}>0$ for all $i$ (i.e. that individuals can leave every class either through mortality or moving to the next class). 
Combining the times in Eq. (B.5) with the rates $\rho_{i}=b_{1 i}-e_{i i}$ in the form of the fitness in Eq. (B.3) gives

$$
s_{x}(y) \propto \sum_{i=1}^{n}\left(b_{1 i}-e_{i i}\right) \frac{\prod_{j=0}^{i-1}\left(t_{\{j+1\} j}\right)}{\prod_{j=1}^{i}\left(t_{\{j+1\} j}+e_{j j}\right)} .
$$

If we omit a positive common denominator, $\prod_{j=1}^{n+1}\left(t_{\{j+1\} j}+e_{j j}\right)$ where $\left(t_{\{n+2\}\{n+1\}}+\right.$ $\left.e_{\{n+1\}\{n+1\}}\right)=1$, then we can write the fitness as

$$
s_{x}(y) \propto \sum_{i=1}^{n}\left[\left(b_{1 i}-e_{i i}\right)\left(\prod_{j=0}^{i-1} t_{\{j+1\} j}\right)\left(\prod_{j=i+1}^{n+1}\left(t_{\{j+1\} j}+e_{j j}\right)\right)\right] .
$$

It is this form for the fitness (also shown in Eq. (5)) that we use to prove the three criteria introduced in the main text.

We later relax some of the assumptions underlying Eq. (B.7). With such generalisations in mind, it is worth observing that Eq. (B.7) already includes the case of sequential movement through classes but with $t_{n} \neq 0$ so that returning to the initial class is possible as in some infectious models with recovery. Phenomenologically this can be established by regarding Eq. (B.4) as giving the time for the first pass $T_{i}^{(1)}$. After returning to class 1 , the individuals move through the classes for a second time (and subsequently a third and fourth time and so on) during which the average time spent in each class will be $T_{i}^{(2)}$ (and $T_{i}^{(3)}, T_{i}^{(4)}$ and so on). The total average time an individual will spend in each class will be $T_{i}=\sum_{j=1}^{\infty} T_{i}^{(j)}$ (i.e. the sum of the times it spends in class $i$ during each pass through). However, this is the sum of a geometric series and each total takes the form $T_{i}=\sum_{j} T_{i}^{(j)}=A T_{i}^{(1)}$, where $A$ is the same (positive) factor for all $i$. Hence the fitness, which takes the form $s_{x}(y) \propto \sum_{i} \rho_{i} T_{i}$ can be written as $s_{x}(y) \propto A \sum_{i} \rho_{i} T_{i}^{(1)}$. Omitting the (positive) constant $A$, we can take the times $T_{i}^{(1)}$ as our $T_{i}$ for the fitness, omitting $A$ from further 
calculations. Formally, $\mathbf{- G T}=\mathbf{I}$ gives $n-1$ equations for the ratios $T_{i} / T_{1}$ which correspond to those derived from Eq. (B.4). Although $T_{1}$ is not as at Eq. (B.4), it may be omitted from Eq. (B.3) by removing it as a positive factor leaving an expression in the above ratios.

B.2 Criterion A - The evolving parameters only appears in the dynamics describing class $k$, where $k \neq 1$

In section 3.1.1 we showed that if the evolving parameters only appear in the dynamics describing a single class and that was class 1 , then the invasion boundaries would always be linear and superimposed. Here we again take the evolving parameters to appear in the dynamics describing a single class, but this time it is not class 1 .

Again we aim to show that $\partial X_{i} /\left.\partial x\right|_{x^{*}}=0$ for all $i$, however, in this case, the evolving parameters can only exist in the mortality terms, $e_{i}$ (where $i \neq 1$ ) - these appear in the dynamics describing one class only. Let us assume that this is class $k$, where $k \neq 1$, and hence only in $e_{k k}$.

Focussing on the resident dynamics for the class $k$, we find that Eq. (7) is replaced by

$$
\sum_{j}\left(\frac{\partial F_{k}}{\partial X_{j}}\right) \frac{d X_{j}}{d x}-\frac{\partial e_{k k}}{\partial x} X_{k}=0
$$

Differentiating the fitness in Eq. (B.7) with respect to the mutant invader $y$ and evaluating at the evolutionary singularity, gives 


$$
\begin{aligned}
\left.\frac{\partial s_{x}(y)}{\partial y}\right|_{x^{*}} \propto & \left.\frac{\partial e_{k k}}{\partial y}\right|_{x^{*}}\left[-\left(\prod_{j=0}^{k-1} t_{\{j+1\} j}\right)\left(\prod_{j=k+1}^{n+1}\left(t_{\{j+1\} j}+e_{j j}\right)\right)\right. \\
+ & \sum_{i=1}^{k-1}\left(b_{1 i}-e_{i i}\right)\left(\prod_{j=0}^{i-1} t_{\{j+1\} j}\right)\left(\prod_{j=i+1}^{k-1}\left(t_{\{j+1\} j}+e_{j j}\right)\right) \\
& \left.\times\left(\prod_{j=k+1}^{n+1}\left(t_{\{j+1\} j}+e_{j j}\right)\right)\right]\left.\right|_{x^{*}}=0,
\end{aligned}
$$

since only $e_{k k}$ is taken to vary as $y$ changes. Using the form for the densities in Eq. (10) this can be re-written as

$$
\begin{array}{r}
\left.\frac{\partial e_{k k}}{\partial y}\right|_{x^{*}}\left(\left.\prod_{j=k+1}^{n+1}\left(\bar{t}_{\{j+1\} j}+\bar{e}_{j j}\right)\right|_{x^{*}}\right)\left[-\left(\frac{X_{k}}{X_{1}} \prod_{j=2}^{k}\left(\bar{t}_{\{j+1\} j}+\bar{e}_{j j}\right)\right)\right. \\
\left.+\sum_{i=1}^{k-1}\left(\bar{b}_{1 i}-\bar{e}_{i i}\right) \frac{X_{i}}{X_{1}}\left(\prod_{j=2}^{k-1}\left(\bar{t}_{\{j+1\} j}+\bar{e}_{j j}\right)\right)\right]\left.\right|_{x^{*}}=0 .
\end{array}
$$

With some simplifying, this becomes

$$
\begin{array}{r}
\left.\frac{\partial e_{k k}}{\partial y}\right|_{x^{*}} \frac{1}{X_{1}}\left(\left.\prod_{j=2}^{k-1}\left(\bar{t}_{\{j+1\} j}+\bar{e}_{j j}\right)\right|_{x^{*}}\right)\left(\left.\prod_{j=k+1}^{n+1}\left(\bar{t}_{\{j+1\} j}+\bar{e}_{j j}\right)\right|_{x^{*}}\right) \\
{\left.\left[-X_{k}\left(\bar{t}_{\{k+1\} k}+\bar{e}_{k k}\right)+\sum_{i=1}^{k-1}\left(\bar{b}_{1 i}-\bar{e}_{i i}\right) X_{i}\right]\right|_{x^{*}}=0}
\end{array}
$$

Using the fact that $\bar{e}_{i i} X_{i}=\bar{t}_{i\{i-1\}} X_{i-1}-\bar{t}_{\{i+1\} i} X_{i}$ (see Eq. (9)) and that $X_{1}>0$ and $\bar{t}_{\{i+1\} i}+\bar{e}_{i i}>0$ for all $i$, gives

$$
\begin{aligned}
\left.\frac{\partial e_{k k}}{\partial y}\right|_{x^{*}} & {\left[-X_{k}\left(\bar{t}_{\{k+1\} k}+\bar{e}_{k k}\right)+\bar{t}_{k\{k-1\}} X_{k-1}\right.} \\
& \left.-\bar{t}_{21} X_{1}-\bar{e}_{11} X_{1}+\sum_{i=1}^{k-1} \bar{b}_{1 i} X_{i}\right]\left.\right|_{x^{*}}=0 .
\end{aligned}
$$

Again using the equilibrium conditions $\bar{e}_{k k} X_{k}=\bar{t}_{k\{k-1\}} X_{k-1}-\bar{t}_{\{k+1\} k} X_{k}$ and that derived from $d X_{1} / d t=0$, simplifies this to

$$
\left.\left.\frac{\partial e_{k k}}{\partial y}\right|_{x^{*}}\left[-\bar{t}_{1 n} X_{n}-\sum_{i=k}^{n} \bar{b}_{1 i} X_{i}\right]\right|_{x^{*}}=0
$$


Thus

$$
\left.\frac{\partial e_{k k}}{\partial y}\right|_{x^{*}}=0
$$

and Eq. (B.8) simplifies at the singularity to give

$$
A_{k 1} \frac{d X_{1}}{d x}+\ldots+A_{k m} \frac{d X_{m}}{d x}=0
$$

The remainder of the proof showing that the two invasion boundaries are superimposed and linear is identical to that for the previous case, in section 3.1.1.

\section{Relaxing the technical assumptions}

\section{C.1 Individuals enter the system through any class}

Here we calculate the fitness and determine whether the three criteria still hold when we relax the constraint limiting which class individuals can be born into.

Earlier we noted that the fitness can be found by a number of methods. Elsewhere here we have used a census of the population in the next generation following an invasion. This method is equivalent to identifying the fitness with $R_{0}-1$ where $R_{0}$ is the maximum eigenvalue of the next generation matrix (Diekmann and Heesterbeek, 2000). When we relax the assumption regarding reproduction, this is computationally difficult; hence here we prefer to use $r$, the maximum eigenvalue of the invasion Jacobian (Metz et al., 1996a; Geritz et al., 1998).

Calculating the Jacobian matrix for the mutant dynamics above, in Eq. (19) 
and evaluating each entry at the equilibrium for the established resident existing alone (i.e. with $Y_{1}=Y_{2}=0$ ) gives

$$
\mathbf{J}=\left(\begin{array}{cc}
b_{11}-e_{11}-t_{21} & b_{12}+t_{12} \\
b_{21}+t_{21} & b_{22}-e_{22}-t_{12}
\end{array}\right) \text {. }
$$

The off-diagonal elements of $\mathbf{J}$ are positive; hence we have two real eigenvalues. The entries on the main diagonal are negative (due to the equilibrium conditions of $\left(\bar{b}_{11}-\bar{e}_{11}-\bar{t}_{21}\right) X_{1}=-\left(\bar{b}_{12}+\bar{t}_{12}\right) X_{2}$ etc. $)$; hence so is the trace of this matrix and the minimum eigenvalue. Thus $r$ and $-\operatorname{det}(\mathbf{J})$ are sign equivalent and we can use the latter as a substitute fitness so

$$
s_{x}(y) \propto-\left(b_{11}-e_{11}-t_{21}\right)\left(b_{22}-e_{22}-t_{12}\right)+\left(b_{12}+t_{12}\right)\left(b_{21}+t_{21}\right) .
$$

Using this form we go on to examine whether the three criteria hold when the assumption as to which class newborn individuals enter is relaxed.

\section{C.1.1 Criterion A: the appearing criterion}

First we consider criterion A (appearing) and assume this criterion is not satisfied, hence all the evolving parameters appear in the dynamics describing a single class only, which, because of symmetry, we take without loss of generality to be class 1 . The evolving parameters can only appear in the terms $b_{1 i}$ and $e_{11}$. The analogue of Eq. (8) is

$$
\begin{array}{r}
\left.\frac{\partial s_{x}(y)}{\partial y}\right|_{x^{*}} \propto-\left(\left.\frac{\partial b_{11}}{\partial y}\right|_{x^{*}}-\right. \\
\left.-\left.\frac{\partial e_{11}}{\partial y}\right|_{x^{*}}\right)\left(\bar{b}_{22}-\bar{e}_{22}-\bar{t}_{12}\right) \\
+\left.\frac{\partial b_{12}}{\partial y}\right|_{x^{*}}\left(\bar{b}_{21}+\bar{t}_{21}\right)=0 .
\end{array}
$$


Using the equilibrium derived from the dynamics describing class 2 (i.e. $\left(\bar{b}_{21}+\right.$ $\left.\left.\bar{t}_{21}\right) X_{1}+\left(\bar{b}_{22}-\bar{e}_{22}-\bar{t}_{12}\right) X_{2}=0\right)$ this can be written as

$$
\left.\frac{\partial s_{x}(y)}{\partial y}\right|_{x^{*}} \propto-\left(\bar{t}_{21}+\bar{b}_{21}\right)\left(\left(\left.\frac{\partial b_{11}}{\partial y}\right|_{x^{*}}-\left.\frac{\partial e_{11}}{\partial y}\right|_{x^{*}}\right) \frac{X_{1}}{X_{2}}+\left.\frac{\partial b_{12}}{\partial y}\right|_{x^{*}}\right)=0
$$

As this derivative is zero at the evolutionary singularity and assuming $\bar{t}_{21}+$ $\bar{b}_{21}>0$ (i.e. that individuals from class 1 can move to or create offspring into class 2), we get the equality

$$
\left.\frac{\partial b_{11}}{\partial y}\right|_{x^{*}} X_{1}+\left.\frac{\partial b_{12}}{\partial y}\right|_{x^{*}} X_{2}=\left.\frac{\partial e_{11}}{\partial y}\right|_{x^{*}} X_{1}
$$

Returning to the equilibrium condition for class 1, from Eq. (18), we find

$$
\begin{array}{r}
\sum_{i=1}^{m} \frac{\partial}{\partial X_{i}}\left(\bar{b}_{11} X_{1}+\bar{b}_{12} X_{2}-\bar{e}_{11} X_{1}-\bar{t}_{21} X_{1}+\bar{t}_{12} X_{2}\right) \frac{d X_{i}}{d x} \\
+\left(\frac{\partial \bar{b}_{11}}{\partial x}-\frac{\partial \bar{e}_{11}}{\partial x}\right) X_{1}+\frac{\partial \bar{b}_{12}}{\partial x} X_{2}=0 .
\end{array}
$$

Evaluating this as the evolutionary singularity, and using Eq. (C.5), we can write this as

$$
\left.A_{11} \frac{d X_{1}}{d x}\right|_{x^{*}}+\left.A_{12} \frac{d X_{2}}{d x}\right|_{x^{*}}+\ldots+\left.A_{1 m} \frac{d X_{m}}{d x}\right|_{x^{*}}=0
$$

The remainder of the argument parallels that in section 3.1.1 - hence the criterion holds.

\section{C.1.2 Criterion B: the characteristic criterion}

Focussing on those terms which are characteristic of class 1 (without loss of generality) in the fitness in Eq. (C.2) (the terms with second subscript being $1)$, the rates $b_{1 i}, e_{11}$ and $t_{21}$ again appear linearly. Therefore the invasion boundary $f_{1}$ is again straight. Hence this criterion holds. 


\section{C.1.3 Criterion $C$ : density dependent rates criterion}

The argument we introduced in section 3.3 also holds when we allow newborn individuals to enter the system through any class, hence this criterion holds.

\section{C.2 Individuals can move between classes in any order}

Here we calculate the fitness and determine whether the three criteria still hold when we relax the constraint stating that individuals move through the classes sequentially.

To calculate the fitness we use the approach as earlier, in Eq. (B.3); however relaxing the present assumption makes the average time a mutant individual spends in each class more complicated. To find the average times we must now turn to a more general approach involving the transition matrix $\mathbf{G}$ (Reade et al., 1998; Diekmann and Heesterbeek, 2000). The elements of this matrix, $\mathbf{G}_{i j}$, are the net rates of increase in individuals of class $i$ per individual in class $j$ (excluding birth terms). For the explicit model in Eq. (21), we have

$$
\mathbf{G}=\left(\begin{array}{ccc}
-e_{11}-t_{21}-t_{31} & t_{12} & t_{13} \\
t_{21} & -e_{22}-t_{12}-t_{32} & t_{23} \\
t_{31} & t_{32} & -e_{33}-t_{13}-t_{23}
\end{array}\right) .
$$

The average times $T_{i j}$ which an individual born in class $j$ spends in class $i$, are identified as the elements of $-\mathbf{G}^{-1}$ and hence 


$$
-\mathbf{G}^{-1}=-\frac{1}{|\mathbf{G}|}\left(\begin{array}{cc}
\left(e_{22}+t_{21}+t_{23}\right)\left(e_{33}+t_{31}+t_{32}\right)-t_{23} t_{32} & \ldots \\
t_{13} t_{32}+t_{12}\left(e_{33}+t_{31}+t_{32}\right) & \ldots \ldots \\
t_{12} t_{23}+t_{13}\left(e_{22}+t_{21}+t_{23}\right) & \ldots \ldots
\end{array}\right)
$$

where $|\mathbf{G}|<0$. As all individuals enter the system into class 1, we only require the times that appear in the first column of this matrix. Combining the times in Eq. (C.9) with the rates $\rho_{i}=b_{1 i}-e_{i i}$, gives the fitness as

$$
\begin{aligned}
s_{x}(y) \propto & \left(b_{11}-e_{11}\right)\left(\left(e_{22}+t_{2}\right)\left(e_{33}+t_{3}\right)-t_{23} t_{32}\right) \\
+ & \left(b_{12}-e_{22}\right)\left(e_{33} t_{21}+t_{1} t_{3}-t_{13} t_{31}\right) \\
+ & \left(b_{13}-e_{33}\right)\left(e_{22} t_{31}+t_{1} t_{2}-t_{12} t_{21}\right)
\end{aligned}
$$

where $t_{1}=t_{21}+t_{31}, t_{2}=t_{12}+t_{32}$ and $t_{3}=t_{13}+t_{23}$ and we have dropped the positive factor $-1 /|\mathbf{G}|$.

\section{C.2.1 Appearing criterion}

We aim to establish the result in Eq. (17) in this new context and hence use Eq. (6) to show that the invasion boundaries are superimposed.

We now assume that the evolving parameters only appear in the dynamics describing a single class. First we assume that this is class 1; then only rates $b_{1 i}$ (for $\left.i=1,2,3\right)$ and $e_{11}$ change as these parameters change. Hence

$$
\left.\left.\frac{\partial s_{x}(y)}{\partial y}\right|_{x^{*}} \propto\left(\frac{\partial b_{11}}{\partial y}-\frac{\partial e_{11}}{\partial y}+\frac{\partial b_{12}}{\partial y} \frac{T_{2}}{T_{1}}+\frac{\partial b_{13}}{\partial y} \frac{T_{3}}{T_{1}}\right)\right|_{x^{*}}=0
$$

where we have returned to the form $T_{i}$ for the times for simplicity (as these do not contain $b_{1 i}$ or $e_{11}$ ). Returning to the resident dynamics in Eq. (20) at equilibrium we get 


$$
\begin{aligned}
& X_{2}\left(\bar{e}_{22}+\bar{t}_{2}\right)=\bar{t}_{21} X_{1}+\bar{t}_{23} X_{3}, \\
& X_{3}\left(\bar{e}_{33}+\bar{t}_{3}\right)=\bar{t}_{31} X_{1}+\bar{t}_{32} X_{2} .
\end{aligned}
$$

Rearranging these, we can write the (ratios of) equilibria in terms of the average times spent in each class as

$$
\frac{X_{2}}{X_{1}}=\frac{T_{2}}{T_{1}} \quad \text { and } \quad \frac{X_{3}}{X_{1}}=\frac{T_{3}}{T_{1}}
$$

Eqs. (C.11) and (C.13) give

$$
\left.\left.\frac{\partial s_{x}(y)}{\partial y}\right|_{x^{*}} \propto \frac{\partial b_{11}}{\partial y}\right|_{x^{*}}-\left.\frac{\partial e_{11}}{\partial y}\right|_{x^{*}}+\left.\frac{\partial b_{12}}{\partial y}\right|_{x^{*}} \frac{X_{2}^{*}}{X_{1}^{*}}+\left.\frac{\partial b_{13}}{\partial y}\right|_{x^{*}} \frac{X_{3}^{*}}{X_{1}^{*}}=0 .
$$

Taking the dynamics describing class 1 of the resident strain (set at equilibrium) differentiated through with respect to the resident strain and evaluated at the evolutionary singularity, gives

$$
\begin{array}{r}
\left.\sum_{j=1}^{m} \frac{\partial}{\partial X_{j}}\left(\sum_{i=1}^{3} \bar{b}_{1 i} X_{i}-\bar{e}_{11} X_{1}+\sum_{i=1}^{3}\left(\bar{t}_{1 i} X_{i}-\bar{t}_{i 1} X_{1}\right)\right) \frac{d X_{j}}{d x}\right|_{x^{*}} \\
\left.\sum_{i=1}^{3} \frac{\partial \bar{b}_{1 i}}{\partial x}\right|_{x^{*}} X_{i}-\left.\frac{\partial \bar{e}_{11}}{\partial x}\right|_{x^{*}} X_{1}=0 .
\end{array}
$$

Using Eq. (C.14), this can be simplified as

$$
\left.A_{11} \frac{d X_{1}}{d x}\right|_{x^{*}}+\left.A_{12} \frac{d X_{2}}{d x}\right|_{x^{*}}+\ldots+\left.A_{1 m} \frac{d X_{m}}{d x}\right|_{x^{*}}=0
$$

Using the equations describing the dynamics of classes 2 and 3 for the resident strain and any non-evolving species (all set at equilibrium) we can get similar equations of the form $\sum_{j=1}^{m} A_{i j} d X_{j} / d x=0$, for $i=2, \ldots, m$. The remainder of the argument parallels that in section 3.1.1 - hence this criterion holds.

Secondly we assume that the evolving parameters only appear in the dynamics describing class 2, that is they are confined to the rate term $e_{22}$; Eq. (C.11) is replaced by 


$$
\left.\left.\frac{\partial s_{x}(y)}{\partial y}\right|_{x^{*}} \propto \frac{\partial e_{22}}{\partial y}\right|_{x^{*}}\left[\left(b_{11}-e_{11}\right)\left(e_{33}+t_{3}\right)-T_{2}+\left(b_{13}-e_{33}\right) t_{31}\right]=0(. C .17)
$$

Using the facts that $\sum_{i}\left(b_{1 i}-e_{i i}\right) X_{i}=0$ (found by summing up the resident dynamics at equilibrium in Eq. (20)) and that $T_{2} / T_{1}=X_{2} / X_{1}$ (Eq. (C.13)), this can be rewritten as

$$
\begin{array}{r}
\left.\left.\frac{\partial s_{x}(y)}{\partial y}\right|_{x^{*}} \propto \frac{\partial e_{22}}{\partial y}\right|_{x^{*}} \frac{1}{X_{1}}\left[-\left(b_{12}-e_{22}\right)\left(e_{33}+t_{3}\right) X_{2}\right. \\
\left.-\left(b_{13}-e_{33}\right)\left(e_{33}+t_{3}\right) X_{3}-T_{1} X_{2}+\left(b_{13}-e_{33}\right) t_{31} X_{1}\right]=0 .
\end{array}
$$

Now using the second equality in Eq. (C.12) and the explicit form for $T_{1}$, in Eq. (C.9), this can be simplified to

$$
\begin{gathered}
\left.\left.\frac{\partial s_{x}(y)}{\partial y}\right|_{x^{*}} \propto \frac{\partial e_{2}}{\partial y}\right|_{x^{*}} \frac{1}{X_{1}}\left[-b_{12}\left(e_{33}+t_{3}\right) X_{2}-b_{13} t_{32} X_{2}\right. \\
\left.-e_{33} t_{12} X_{2}-t_{12} t_{13} X_{2}-t_{12} t_{23} X_{2}-t_{32} t_{13} X_{2}\right]=0 .
\end{gathered}
$$

Thus, as all the terms in the square brackets are negative, we find

$$
\left.\frac{\partial e_{22}}{\partial y}\right|_{x^{*}}=0
$$

Thus we have a parallel result to that in Eq. (B.14) - obviously for $e_{33}$ also with the same consequences, namely linear and superimposed invasion boundaries.

\section{C.2.2 Criterion B: the characteristic criterion}

The rates $b_{i j}, e_{i i}$ and $t_{i j}$ are characteristics of class $j$ (the first subscript), and all appear linearly in the fitness (none of these multiply another). Thus, under the assumption that the evolving parameters are also linear in these rates $\left(b_{i j}, e_{i i}\right.$ and $\left.t_{i j}\right)$, the fitness function will be linear in the evolving parameters. 
Hence the invasion boundary $f_{1}$, which stems from $s_{x}(y)$, will be linear. Thus this criterion holds.

C.2.3 Criterion $C$ : density dependent rates criterion

The argument we introduced in section 3.3 also holds if we allow individuals to move arbitrarily between classes, hence this criterion holds. 


\begin{tabular}{|c|c|c|c|c|c|c|}
\hline \multicolumn{3}{|c|}{ Necessary criteria } & $f_{1}$ boundary & $f_{2}$ boundary & Separation & TIP \\
\hline \multicolumn{3}{|c|}{ for each TIP type } & $(x$ resident & ( $y$ resident & of boundaries & type \\
\hline A & $\mathrm{B}$ & $\mathrm{C}$ & $y$ rare) & $x$ rare) & & \\
\hline- & - & - & Straight & Straight & No & $\mathrm{I}$ \\
\hline$\checkmark$ & $\checkmark$ & - & Curved & Curved & No & II \\
\hline$\checkmark$ & - & $\checkmark$ & Straight & Curved & Yes & III \\
\hline$\checkmark$ & $\checkmark$ & $\checkmark$ & Curved & Curved & Yes & IV \\
\hline
\end{tabular}

Table 1: The shape of TIP and the corresponding necessary criterion; see Fig. 1 for the geometrical forms of TIPs I-IV. The criteria A, B and C are the appearing criterion, characteristic criterion and the density dependent rates criterion respectively. 


\begin{tabular}{l|ll} 
& $\lambda_{1} \lambda_{2}>0$ & $\lambda_{1} \lambda_{2}<0$ \\
\hline$\lambda_{2} f^{\prime \prime}<\lambda_{2} f_{1}^{\prime \prime}$ & Attractor & Repellor \\
$\lambda_{2} f_{1}^{\prime \prime}<\lambda_{2} f^{\prime \prime}<\lambda_{2} \frac{1}{2}\left(f_{1}^{\prime \prime}+f_{2}^{\prime \prime}\right)$ & Branching point & Garden of Eden point \\
$\lambda_{2} f^{\prime \prime}>\lambda_{2} \frac{1}{2}\left(f_{1}^{\prime \prime}+f_{2}^{\prime \prime}\right)$ & Repellor & Attractor
\end{tabular}

Table A.1: Shapes of trade-off (in relation to the invasion boundaries $f_{1}$ and $f_{2}$ ) required to production each evolutionary outcome, given a particular sign of $\lambda_{1}$ and $\lambda_{2}$. Here $\lambda_{1}=\operatorname{sign}\left(s_{x^{*}}(y)\right)$ just above the invasion boundary $f_{1}$, $\lambda_{2}=\operatorname{sign}\left(\partial^{2} f_{2} /\left.\partial y^{2}\right|_{x^{*}}-\partial^{2} f_{1} /\left.\partial y^{2}\right|_{x^{*}}\right)$ and $f_{i}^{\prime \prime}=\partial^{2} f_{i} /\left.\partial y^{2}\right|_{x^{*}}$ for $n=1,2$. 


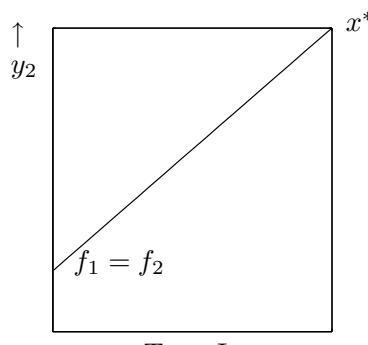

Type I

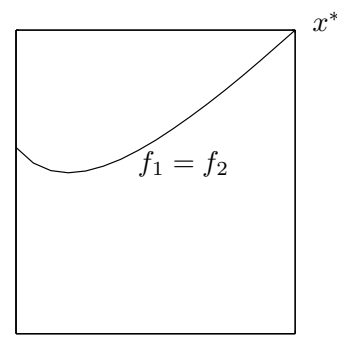

Type II

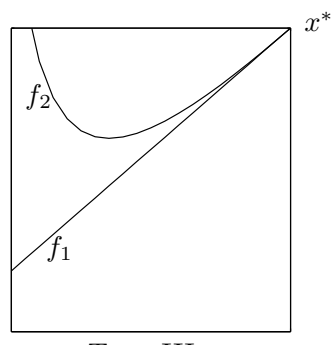

Type III

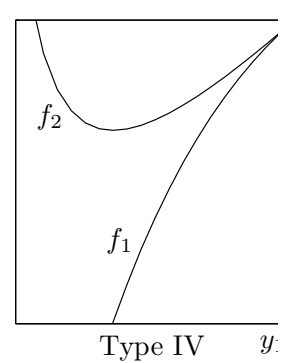

Fig. 1: The four fundamental types of TIP, where type I corresponds to two straight superimposed boundaries, type II to two curved superimposed boundaries, type III to one curved and one straight boundary and type IV to two curved separated boundaries. The combinations of our three criteria which are necessary for these are given in Table $1 .\left(f_{1}\right.$ is the invasion boundary for (mutant) $y$ with $x$ resident; $f_{2}$ for (mutant) $x$ with $y$ resident.) 


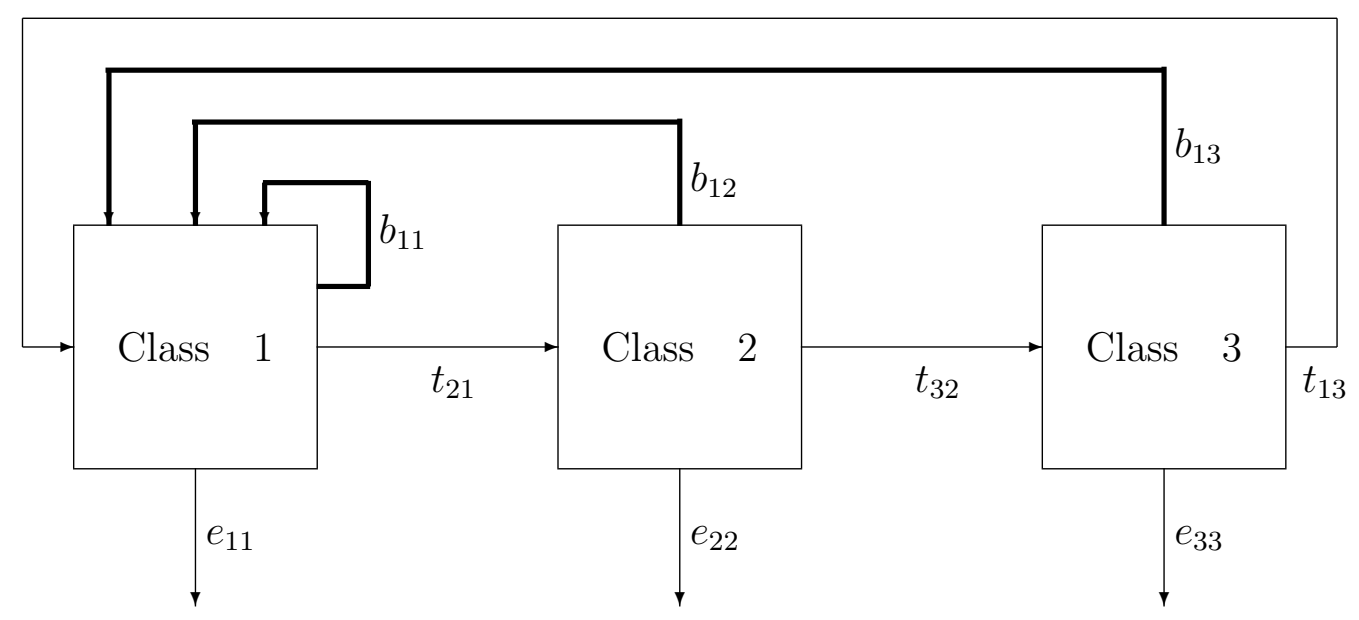

Fig. 2: Life cycle for our system made up of 3 classes, subject to the assumptions that all individuals are born into class 1 (where births are indicated by thick lines) at a rate $b_{1 i}$, individuals move through the classes sequentially, possibly returning to class 1 from the last class, at rates $t_{\{i+1\} i}$. In addition mortality rates for each clas are given by $e_{i i}$. 


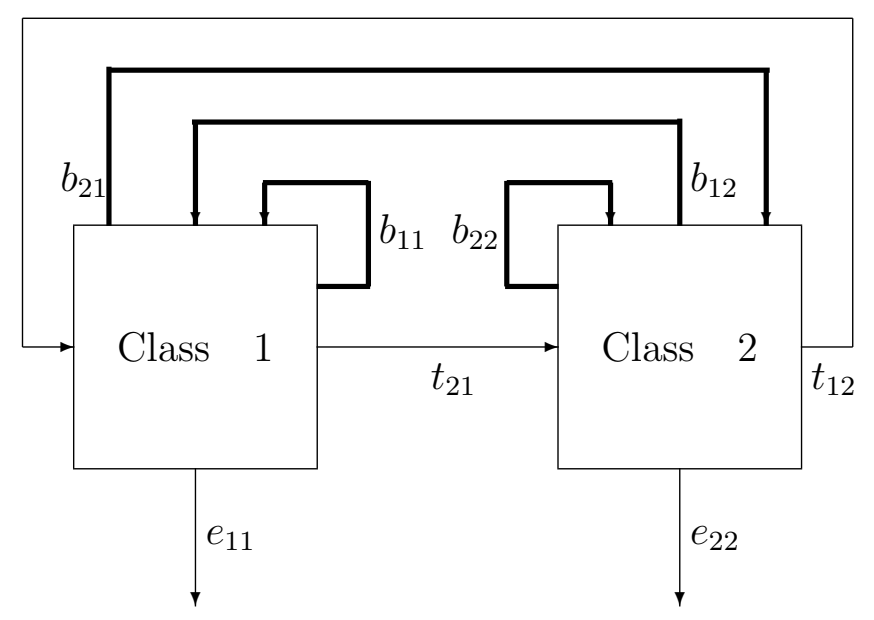

Fig. 3: Life cycle for a system made up of 2 classes (where births are indicated by thick lines at a rate $b_{i j}$ - rate at which individuals in class $j$ give birth to individuals in class $i$ ). The assumption stating that all individuals are born into class 1 has now been relaxed. In addition mortality rates for each class are given by $e_{i i}$ and transition rate by $t_{i j}$. 


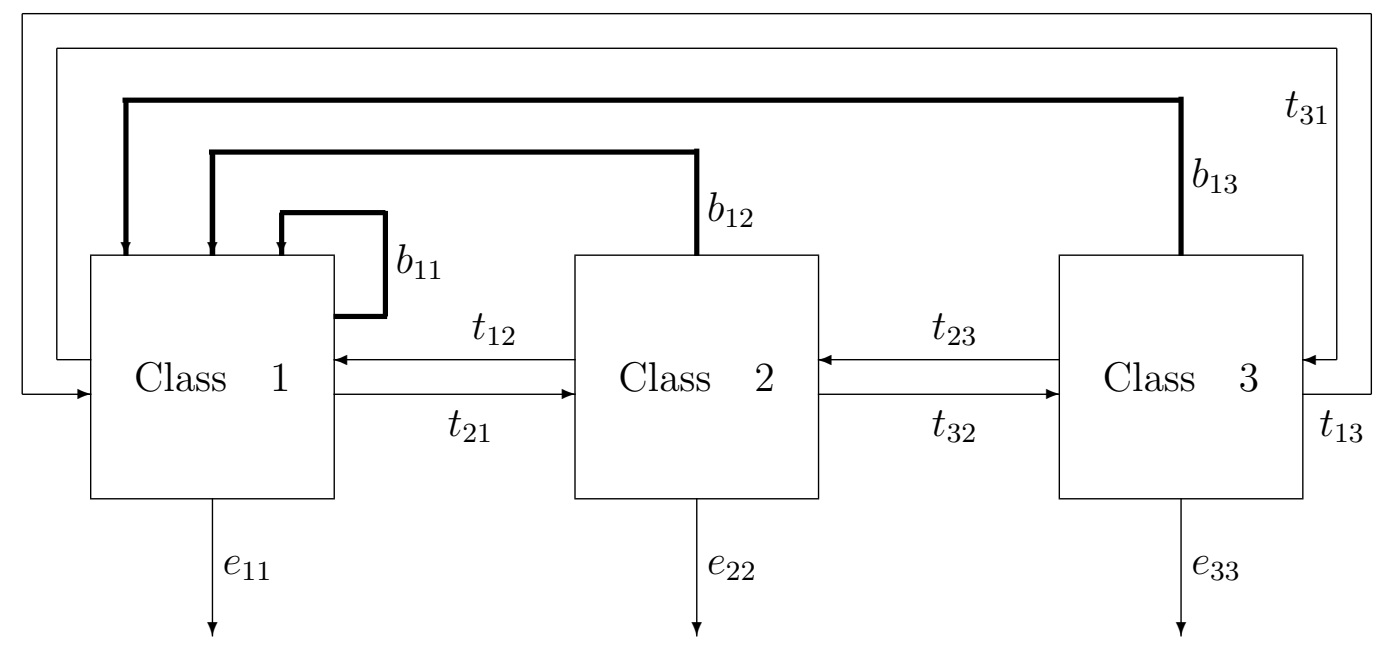

Fig. 4: Life cycle for a system made up of 3 classes, subject to the assumption that all individuals are born into class 1 (where births are indicated by thick lines) at a rate $b_{i j}$. The assumption stating that individuals move through the classes sequentially has now been relaxed and they move from class $j$ to class $i$ at a rate $t_{i j}$. In addition mortality rates for each class are given by $e_{i i}$. 

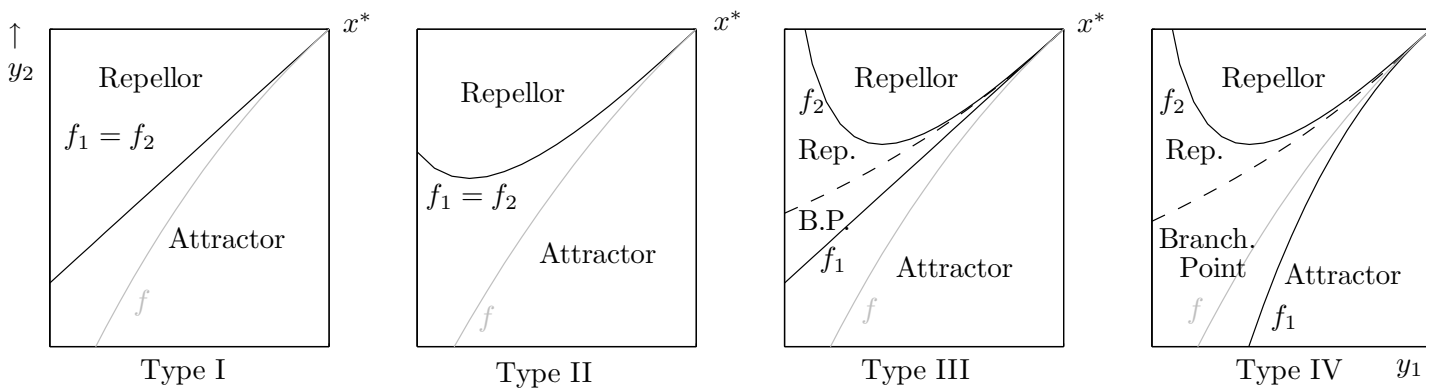

Fig. 5: An example of each of the four types of TIP with the evolutionary outcomes for each region given, with the outcome occuring being determined by which region the trade-off, $f$, enters. In each TIP here the trade-off is a weakly acceleratingly costly trade-off and the evolutionary singularity, $x^{*}$, is an attractor for the type I, type II and type III TIPs, and a branching point for the type IV TIP. ( $f_{1}$ is the invasion boundary for (mutant) $y$ with $x$ resident; $f_{2}$ for (mutant) $x$ with $y$ resident; the 'dashed' curve represents the mean curvature of $f_{1}$ and $f_{2}$.) 